\title{
THE AXIOMS OF SUPERMANIFOLDS AND A NEW STRUCTURE ARISING FROM THEM
}

\author{
MITCHELL J. ROTHSTEIN
}

\begin{abstract}
An analysis of supermanifolds over an arbitrary graded-commmutative algebra is given, proceeding from a set of axioms the first of which is that the derivations of the structure sheaf of a supermanifold are locally free. These axioms are satisfied not by the sheaf of $G^{\infty}$ functions, as has been asserted elsewhere, but by an extension of this sheaf. A given $G^{\infty}$ manifold may admit many supermanifold extensions, and it is unknown at present whether there are $G^{\infty}$ manifolds that admit no such extension. When the underlying graded-commutative algebra is commutative, the axioms reduce to the Berezin-Kostant supermanifold theory.
\end{abstract}

Introduction. When supermanifolds were introduced, the goal was to incorporate anticommuting coordinates in the structure sheaf of a manifold. Yet the constants in this structure sheaf were taken to be $\mathbf{R}$, which has no anticommuting part. Thus the manifold and the supermanifold could not coincide - the supermanifold had to be strictly bigger. Later theories have attempted to bring the manifold and the supermanifold together by introducing an auxiliary Grassmann algebra. Following Rogers [11], fix an infinite set of letters $\beta^{1}, \beta^{2}, \ldots$ Let $B_{L}$ denote the real Grassmann algebra generated by $\beta^{1}, \ldots, \beta^{L}$. If $L=\infty, B_{L}$ is given a suitable Banach norm, making $B_{\infty}$ a Banach-Grassmann algebra as defined in [7]. Rogers refers to supermanifolds based on $B_{L}$ or $B_{\infty}$ as $G^{\infty}$ supermanifolds. [11] deals chiefly with supermanifolds over $B_{L}$ for finite $L$, though it is noted that the case $L=\infty$ would have a greater chance of being relevant to physics. [7] gives an accurate treatment of the infinite dimensional case. Further results regarding the case of finite $L$ may be found in [4 and 10].

A key error was made in [11] and again in [4], the resolution of which has led to the present work. Proposition 5.8 of [11] asserted that the derivations of the structure sheaf of a $G^{\infty}$ manifold form a locally free module over that sheaf. However, the following proposition is valid:

Let $M$ be a $G^{\infty}$ manifold based on $B_{L}$. If Der $\mathscr{G}_{M}^{\infty}$ is a locally free $\mathscr{G}_{M}^{\infty}$ module, then $L=0$ or $\infty$.

Specifically, if $\theta^{1}, \ldots, \theta^{q}$ are odd coordinates on a $G^{\infty}$ manifold, $\partial / \partial \theta^{\alpha}$ does not exist as an operator on $G^{\infty}$ functions. In [4] this difficulty was recognized, and an attempt was made to recover a locally free tangent sheaf by retaining $G_{M}^{\infty}$ as the structure sheaf while redefining "derivation". The redefinition was inconsistent and led again to a false conclusion, Proposition 6.4.

Received by the editors February 21, 1985 and, in revised form, August 23, 1985.

1980 Mathematics Subject Classification. Primary 58A50. 
These errors attest to the desirability of a well-behaved tangent sheaf. In fact, along with free local expression of vector fields as sums of partial derivatives, one may easily list a few key properties which would insure that the geometry of supermanifolds developed in a natural and well-founded way. With enough such properties, one would soon arrive at an axiomatic definition of the desired objects.

This paper will present four such properties, which are offered as the axioms of supermanifolds over an arbitrary graded-commutative algebra $B$. If $B$ is commutative, the axioms reduce to the graded- or supermanifold theory of Berezin [3] and Kostant [8]. If $B=B_{\infty}$, the Banach-Grassmann manifolds of Jadczyk and Pilch [7] are obtained. Though the axioms are meant in part to achieve such a unification and to accent the salient features of supermanifolds, they would perhaps be superfluous were it not that they also produce a nontrivial category of supermanifolds over $B_{L}$ for $0<L<\infty$. The supermanifolds over $B_{L}$ are discussed in $\S 4$. What emerges is Rogers' theory with an added structure. Just as for the case $L=0$, the structure sheaf of the supermanifold is an auxiliary sheaf strictly larger than the sheaf of $G^{\infty}$ functions. When $L$ exceeds the odd dimension of the $G^{\infty}$ manifold $M$, one discovers that there is a sheaf $\mathscr{S}$ of $G^{\infty}$-modules and a class $c[M] \in H^{2}(M, \mathscr{S})$ associated canonically to the $\mathscr{G}^{\infty}$-structure, such that $c[M]=0$ if and only if $M$ admits a supermanifold extension. If $c[M]=0$ the inequivalent supermanifold extensions over $M$ form an affine space, $H^{1}(M, \mathscr{S})$. It remains to be determined whether there are any $G^{\infty}$ manifolds for which $c[M] \neq 0$. In $\S 5$ it is shown that there are examples for which $H^{1}(M, \mathscr{S}) \neq 0$.

A note on terminology: The term " $G$ supermanifold" is generally applied to manifolds based on $B_{L}$. In the present work, the axioms for a supermanifold are presented without reference to a specific graded commutative algebra. Therefore the term "supermanifold" finds itself applied to the $G^{\infty}$ manifold together with its enlarged structure sheaf $\mathscr{A}$. To avoid confusion, the term " $G^{\infty}$ manifold", will be used for the manifold itself. The structure sheaf of $M$ will be called $\mathscr{G}$, and if $\mathscr{A}$ and $\mathscr{G}$ do not coincide, $\mathscr{A}$ will be called a supermanifold extension of $\mathscr{G}$.

Much of this work was part of the author's Ph.D. thesis, and the author is pleased to acknowledge the help and guidance of his advisor, Robert Blattner. Thanks are also due the referee for many useful suggestions.

0. Graded objects. In this work, the word graded will mean $\mathbf{Z}_{2}$-graded.

A graded space is a vector space $V$ with a decomposition $V=V_{0} \oplus V_{1} . V_{0}$ is called the even part of $V$ and $V_{1}$ is called the odd part of $V$. Elements of $V_{i}$ have parity $i$ and the parity of $v$ is denoted by $|v|$.

If $A$ and $B$ are graded spaces, $A \oplus B$ is graded by

$$
(A \oplus B)_{i}=A_{i} \oplus B_{i} \text {. }
$$

$A \otimes B$ is graded by

$$
(A \otimes B)_{i}=\sum_{j+k=i} A_{j} \otimes B_{k}
$$

$\operatorname{Hom}(A, B)$ is graded by requiring that the natural map $\operatorname{Hom}(A, B) \otimes A \rightarrow B$ be even. 
If $B$ is graded space and also an algebra, $B$ is called a graded algebra if multiplication is an even element of $\operatorname{Hom}(B \otimes B, B)$. An associative graded algebra $B$ is called graded commutative if

$$
a b=(-1)^{|a||b|} b a
$$

when $a$ and $b$ is homogeneous.

If $B$ is an associative graded algebra, a left graded module over $B$ is a left $B$ module such that $M$ is graded, and scalar multiplication is an even element of $\operatorname{Hom}(B \otimes M, M)$. If $B$ is graded commutative, $M$ becomes a right $B$ module by defining

$$
m b=(-1)^{|b||m|} b m, \quad \text { for } b \in B \text { and } m \in M .
$$

$M$ is called $(p, q)$ dimensional if $M$ is free over $B$, with $p$ even and $q$ odd generators. Such a set of generators is called a graded basis for $M$.

If $M$ and $N$ are left $B$ modules, the set of $B$ linear maps from $M$ to $N$ is not the best object to consider. Better is to consider graded linear maps. If $\sigma$ is a homogeneous real linear map from $M$ to $N$, say $\sigma$ is graded linear if

$$
\sigma(b m)=(-1)^{|b||\sigma|} b \sigma(m),
$$

for $b \in B$ and $m \in M$. Denote the graded linear maps by $\operatorname{Hom}_{B}(M, N)$, or $\operatorname{Hom}(M, N)$ if $B$ is understood. Then:

0.1 . $\operatorname{Hom}(M, N)$ is a left $B$ module.

0.2. The action of $B$ on $M$ defines an imbedding $B \rightarrow \operatorname{Hom}(M, M)$, making $\operatorname{Hom}(\boldsymbol{M}, \boldsymbol{M})$ an algebra over $B$.

0.3. If $M$ is free with $n$ generators, then $\operatorname{Hom}(M, M)$ is isomorphic to the $n \times n$ matrices with entries in $B$.

0.4. $\operatorname{Hom}(M, N)$ is the same as $B$-right-linear homomorphisms.

Indeed, if $\sigma \in \operatorname{Hom}(M, N)$ then

$$
\sigma(m b)=(-1)^{|b||m|} \sigma(b m)=(-1)^{|b||\sigma(m)|} b \sigma(m)=\sigma(m) \cdot b .
$$

If $M$ and $N$ are left graded $B$ modules, there are various Cartesian products that can be formed from $M$ and $N$. For $i=0,1$ and $j=0,1$, we define the $(i, j)$ Cartesian product of $M$ and $N$ to be a left graded $B$ module $A$, with graded linear maps $\pi_{1}: A \rightarrow M, \pi_{2}: A \rightarrow N$, where $\left|\pi_{1}\right|=i$ and $\left|\pi_{2}\right|=j$, such that given any left graded $B$ module $C$ and maps $\sigma: C \rightarrow M, \tau: C \rightarrow N$, such that $|\sigma|=i$ and $|\tau|=j$, there exists a unique even map $\sigma \times \tau: C \rightarrow A$ such that

$$
\begin{aligned}
& \text { C }
\end{aligned}
$$

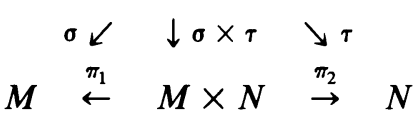

commutes.

(The alternative to this definition is to define the module $R(M)$ by setting $R(M)_{i}=M_{i+1}$ and then take ordinary Cartesian products of $M$ 's and $R(M)$ 's. There are two disadvantages to this. First, in the Cartesian product of $B$ with itself, 
it is preferable that the coordinate functions have values in a graded commutative algebra, which $R(B)$ is not. Second, coordinate functions must be odd on occasion, and in the ordinary Cartesian product they are necessarily even.)

Define $B^{p}$ to be the $(1,0)$ Cartesian product of $B^{p-1}$ and $B$, and define $B^{p / q}$ to be the $(1,1)$ Cartesian product of $B^{p}$ and $B^{q} . B^{p / q}$ is a free $(p, q)$-dimensional $B$ module. As an ungraded Cartesian product, $B^{p / q}$ is $B^{p+q}$ with its usual projection functions. While the first $p$ projections are even maps, the last $q$ are odd.

Definition. $B^{p, q}$ is defined to be the even part of $B^{p / q}$. Thus,

$$
B^{p, q}=\left\{\left(b^{1}, \ldots, b^{p+q}\right)|| b^{i} \mid=0 \text { for } i \leqslant p \text { and }\left|b^{i}\right|=1 \text { for } i>p\right\} .
$$

Note that if $\varepsilon \in B$ and $v=\left(b^{1}, \ldots, b^{p+q}\right) \in B^{p / q}$, then

$$
\pi^{i}(\varepsilon v)=(-1)^{\left|\pi^{i}\right||\varepsilon|} \varepsilon \pi^{i}(v)=(-1)^{\left|\pi^{i}\right||\varepsilon|} \varepsilon b^{i},
$$

so that $\varepsilon$ acts as the matrix,

$$
\left(\begin{array}{cccccc}
\varepsilon & & & & & \\
& & \varepsilon & & & \\
& & (-1)^{|\varepsilon|} \varepsilon & & \\
& & & & (-1)^{|\varepsilon|} \varepsilon
\end{array}\right) .
$$

If $N$ is a submodule of $M, N$ is called a graded submodule if

$$
N=N \cap M_{0} \oplus N \cap M_{1} .
$$

Then $N$ inherits a grading from $M$, and

$$
M / N=M_{0} / N_{0} \oplus M_{1} / N_{1},
$$

so that $M / N$ is also graded, and the maps $0 \rightarrow N \rightarrow M \rightarrow M / N \rightarrow 0$ are even.

1. Basic properties of supermanifolds. To describe supermanifolds, the following objects will be needed.

1. A graded-commutative Banach algebra $B$ over $\mathbf{R}$.

2. A Hausdorff topological space $M$.

3. A sheaf on $M$ of graded-commutative algebras over $B$, called $(M, \mathscr{A})$ or $\mathscr{A}$.

4. An evaluation map $\mathscr{A} \stackrel{\delta}{\rightarrow} \mathscr{C}$ where $\mathscr{C}$ is the sheaf of continuous $B$-valued functions on $M$.

The sections of $\mathscr{A}$ will be referred to as superfunctions.

The axioms presented here are intended to give a workable notion of vector fields on a supermanifold. Given a stalk $\left.\mathscr{A}\right|_{x}, x \in M$, a graded derivation of $\left.\mathscr{A}\right|_{x}$ is a $B$-graded-linear endomorphism $X:\left.\left.\mathscr{A}\right|_{X} \rightarrow \mathscr{A}\right|_{X}$ satisfying

$$
X(f g)=X f \cdot g+(-1)^{|X||f|} f \cdot X g
$$

for $X$ and $f$ homogeneous. Let $\left.\operatorname{Der} \mathscr{A}\right|_{x}$ denote the set of graded derivations of $\left.\mathscr{A}\right|_{x}$. Der $\left.\mathscr{A}\right|_{x}$ inherits a grading from End $\left.\mathscr{A}\right|_{x}$, and Der $\left.\mathscr{A}\right|_{x}$ is a left graded $\left.\mathscr{A}\right|_{x}$ submodule of End $\left.\mathscr{A}\right|_{x}$. The Lie bracket is defined on homogeneous elements of 
End $\left.\mathscr{A}\right|_{x}$ by

$$
[X, Y]=X Y-(-1)^{|X||Y|} Y X
$$

$[$,$] satisfies:$

1.1. $[b X, Y]=b[X, Y], b \in B$.

1.2. $[X, Y]=(-1)^{1+|X||Y|}[Y, X]$.

1.3. $[[X, Y], Z]-[X,[Y, Z]]+(-1)^{|X||Y|}[Y,[X, Z]]=0$.

1.4. $[X, Y Z]=[X, Y] Z+(-1)^{|X||Y|} Y[X, Z]$.

1.5. Der $\left.\mathscr{A}\right|_{x}$ is closed under bracket.

For $U$ an open subset of $M$, let $\operatorname{Der} \mathscr{A}(U)$ be the set of morphisms $X:\left.\left.\mathscr{A}\right|_{U} \rightarrow \mathscr{A}\right|_{U}$ such that the restriction of $X$ to each stalk is a graded derivation. Then Der $\mathscr{A}$ is a complete presheaf, and the corresponding sheaf is denoted by the same name. $\operatorname{Der} \mathscr{A}(U)$ acts on $\mathscr{A}(U)$ by composition, and this action factors through to germs, giving a map $\left.\left.(\operatorname{Der} \mathscr{A})\right|_{x} \rightarrow \operatorname{Der} \mathscr{A}\right|_{x} \mathscr{A}(U)$ acts on $\operatorname{Der} \mathscr{A}(U)$ by $f X(m)=f(m) X(m)$ and this gives a left action of $\left.\mathscr{A}\right|_{x}$ on Der $\left.\mathscr{A}\right|_{x}$. Define Der* $\mathscr{A}(U)$ to be the set of sheaf morphisms $\omega:\left.\left.\operatorname{Der} \mathscr{A}\right|_{U} \rightarrow \mathscr{A}\right|_{U}$ whose restriction to each stalk is an $\left.\mathscr{A}\right|_{x}$-graded-linear transformation. Der* $\mathscr{A}$ is a sheaf of left $\mathscr{A}$ modules. The corresponding right action of $\mathscr{A}$ on $\operatorname{Der}^{*} \mathscr{A}$ is given by $\omega f(X)=$ $\omega(f X)$. For a section $f \in \mathscr{A}(U)$, define the differential $d f \in \operatorname{Der}^{*} \mathscr{A}(U)$ by

$$
d f(X)=(-1)^{|f||X|} X f .
$$

This also factors through to germs, giving a sheaf morphism $d: \mathscr{A} \rightarrow$ Der* $\mathscr{A}$. Since, by definition, $|X f|=|X|+|f|$, one has $|d f|=|f|$. Also

$$
\begin{aligned}
d(f g)(X) & =(-1)^{|f g||X|} X(f g)=(-1)^{|f g||X|}\left(X f \cdot g+(-1)^{|f||X|} f \cdot X g\right) \\
& =(-1)^{|g||f|+|f||X|} g \cdot X f+f \cdot d g(X)=(d f \cdot g+f \cdot d g)(X) .
\end{aligned}
$$

Axiomatic definition of a supermanifold. Let $p$ and $q$ be nonnegative integers. $(M, \mathscr{A}, \delta)$ is a $(p, q)$-dimensional supermanifold with ground ring $B$ if the following four axioms are satisfied.

1. Coordinate systems exist. For any $x \in M$ there exists a neighborhood $U$ of $x$ and superfunctions $\xi^{1}, \ldots, \xi^{p} \in \mathscr{A}_{0}(U)$ and $\xi^{p+1}, \ldots, \xi^{p+q} \in \mathscr{A}_{1}(U)$ such that $\left(d \xi^{1}, \ldots, d \xi^{p+q}\right)$ is a graded basis for $\operatorname{Der}{ }^{*} \mathscr{A}(U)$ over $\mathscr{A}(U)$.

The integers $p$ and $q$ are called the even and odd dimensions, respectively, of $(M, \mathscr{A}, \delta)$. Any set of sections $\left(\xi^{1}, \ldots, \xi^{p+q}\right)$ satisfying axiom 1 is called a coordinate system for $\left.\mathscr{A}\right|_{U}$.

Given $\left.f \in \mathscr{A}\right|_{x}$, denote by $\tilde{f}$ the image of $f$ under $\delta$. $\tilde{f}(x)$ is called the value of $f$ at $x$.

2. $\mathscr{A}$ determines a manifold structure on $M$. If $\left(\xi^{1}, \ldots, \xi^{p+q}\right)$ is a coordinate system in a neighborhood $U$, the map $\tilde{\xi}: U \rightarrow B^{p, q}, x \mapsto\left(\tilde{\xi}^{1}(x), \ldots, \tilde{\xi}^{p+q}(x)\right)$, is a homeomorphism onto an open subset of $B^{p, q}$.

3. Taylor expansions exist. Let $\left(\xi^{1}, \ldots, \xi^{p+q}\right)$ be a coordinate system in a neighborhood $U$. Then for any $x \in U$ and any $\left.g \in \mathscr{A}\right|_{x}$ there exist $g_{1}, \ldots,\left.g_{p+q} \in \mathscr{A}\right|_{x}$ such that

$$
g=\tilde{g}(x)+g_{i} \cdot\left(\xi^{i}-\tilde{\xi}^{i}(x)\right) .
$$

(Summation over repeated upper and lower indices is assumed.) 
4. A superfunction is determined by the values of all its derivatives. Let $\mathscr{D}$ denote the sheaf of differential operators on $\mathscr{A}$. That is, $\mathscr{D} \subset$ End $\mathscr{A}$ is the sheaf of subalgebras generated by $\mathscr{A}$ and Der $\mathscr{A}$. For any $\left.f \in \mathscr{A}\right|_{x}$, if $\widetilde{L f}=0$ for all $\left.L \in \mathscr{D}\right|_{x}$, then $f=0$.

By axiom $1, \operatorname{Der} * \mathscr{A}$ is a locally free $(p, q)$-dimensional $\mathscr{A}$ module. Then so is $\operatorname{Der}^{* *} \mathscr{A}$. In addition to the usual map Der $\mathscr{A} \rightarrow \operatorname{Der}^{* *} \mathscr{A}$, there is also $\operatorname{Der}^{* *} \mathscr{A} \stackrel{\mu}{\rightarrow}$ Der $\mathscr{A}$ defined by $\mu X(f)=X(d f)$. It follows that Der $\mathscr{A}$ and Der** $\mathscr{A}$ are isomorphic. Thus, given a coordinate system $\xi^{1}, \ldots, \xi^{p+q}$, one may define the partial derivatives $\partial / \partial \xi^{1}, \ldots, \partial / \partial \xi^{p+q} \in \operatorname{Der} \mathscr{A}(U)$ by

$$
d \xi^{i}\left(\frac{\partial}{\partial \xi^{j}}\right)=\delta_{j}^{i}
$$

$\left(\partial \xi^{i} / \partial \xi^{j}\right.$ must then be $\left.(-1)^{\left|\xi^{i}\right|\left|\xi^{j}\right|} \delta_{i}^{j}\right)$.

EXAMPLES. 1. Supermanifolds or graded manifolds as defined by Berezin [3] and Kostant [8]. $\delta$ is the augmentation map $\mathscr{A} \rightarrow C^{\infty}$ and $B=\mathbf{R}$.

2. The Banach-Grassmann supermanifolds defined by Jadczyk and Pilch [7]. $\mathscr{A}$ is the sheaf of supersmooth functions and $\delta$ is the identity.

THEOREM 1.1. Let $\xi$ be a coordinate system in a neighborhood $U \subset M$. Then for all $j$ and $k,\left[\partial / \partial \xi^{j}, \partial / \partial \xi^{k}\right]=0$.

Proof. Set $D_{j k}=\left[\partial / \partial \xi^{j}, \partial / \partial \xi^{k}\right]$. For a sequence

$$
s=\left(s_{1}, \ldots, s_{n}\right) \in\{1, \ldots, p+q\}^{n}
$$

define $\partial / \partial \xi^{s}=\partial / \partial \xi^{s_{n}} \ldots \partial / \partial \xi^{s_{1}}$ and define $\xi^{s}=\xi^{s_{1}} \ldots \xi^{s_{n}}$.

Take $f \in \mathscr{A}(U)$ and $x \in U$. One may assume $\tilde{\xi}(x)=0$. Let $|s|=n$. Then for any positive integer $J$, repeated application of axiom 3 yields

$$
f=\sum_{|s|<J} \xi^{s} a_{s}+\sum_{|t|=J} \xi^{t} f_{t}
$$

where the $a_{s}$ 's are constants. Then

$$
\frac{\widetilde{\partial f}}{\partial \xi^{s}}(x)=\sum_{\sigma} \pm a_{\sigma(s)},
$$

where $\sigma$ runs over permutations of $s$, and a minus is introduced when two odd coordinates are transposed. It follows that $\delta \circ \partial / \partial \xi^{s}(f)$ is graded-symmetric in the $\partial / \partial \xi^{i}$ 's. In particular, $\delta \circ \partial / \partial \xi^{s} \circ D_{j k}=0$ for all $s$. Since the $\partial / \partial \xi^{s}$ 's span $\mathscr{D}(U)$ over $\mathscr{A}(U)$, axiom 4 yields $D_{j k}=0$.

Given the coordinate system $\xi$ for $\left.\mathscr{A}\right|_{U}$, let $\left.\hat{\mathscr{A}}_{\xi} \subset \mathscr{A}\right|_{U}$ consist of those germs which are independent of the odd coordinates $\xi^{p+1}, \ldots, \xi^{p+q}$. That is,

$$
\hat{\mathscr{A}}_{\xi}=\left\{\left.f \in \mathscr{A}\right|_{U}: \frac{\partial f}{\partial \xi^{i}}=0, i=p+1, \ldots, p+q\right\} .
$$

Let $\Lambda(\xi)$ denote the exterior algebra generated by the odd coordinates $\xi^{p+1}$, $\ldots, \xi^{p+\mathrm{q}}$. 
In general, neither $\Lambda(\xi)$ nor $\hat{\mathscr{A}}_{\xi}$ are invariant under change of coordinates. However, when $B$ has no odd part, $\hat{\mathscr{A}}_{\xi}$ is independent of coordinates. This will be discussed in $\S 3$.

Let $\mu, \nu, \ldots$ denote multi-indices of the form $\mu=\left(\mu_{1}, \ldots, \mu_{q}\right) \in\{0,1\}^{q}$. Set

$$
\xi^{\mu}=\left(\xi^{p+1}\right)^{\mu_{1}} \cdots\left(\xi^{p+q}\right)^{\mu_{q}} \text { and } \frac{\partial}{\partial \xi^{\mu}}=\left(\frac{\partial}{\partial \xi^{p+q}}\right)^{\mu_{q}} \cdots\left(\frac{\partial}{\partial \xi^{p+1}}\right)^{\mu_{1}} \text {. }
$$

THEOREM 1.2. The multiplication map $\left.\mathscr{A}_{\xi} \otimes \Lambda(\xi) \rightarrow \mathscr{A}\right|_{U}$ is an isomorphism.

Proof. One must show that for any $f \in \mathscr{A}(U)$ there are unique elements $f_{\mu} \in \hat{\mathscr{A}}_{\xi}(U)$ such that $f=f_{\mu} \xi^{\mu}$.

For the uniqueness, let $0=f_{\mu} \xi^{\mu}, f_{\mu} \in \hat{\mathscr{A}}_{\xi}(U)$. From graded commutativity of partial differentiation it follows that for all $\nu$

$$
0=\frac{\partial}{\partial \xi^{\nu}}\left(\xi^{\mu} f_{\mu}\right)= \pm f_{\nu}+\sum_{\rho>\nu} \pm \xi^{\rho-\nu} f_{\rho} .
$$

It then follows by induction on $q-|\mu|$ that $f_{\mu}=0$ for all $\mu$.

For the existence, let $R$ be the subalgebra of $\mathscr{A}(U)$ generated by $\xi^{p+1}, \ldots, \xi^{p+q}$ over $\hat{\mathscr{A}}_{\xi}(U)$. The claim is that $R=\mathscr{A}(U)$. For $j>p$, define

$$
S_{j}=\left\{f \in \mathscr{A}(U) \mid \frac{\partial f}{\partial \xi^{k}}=0 \text { if } j \leqslant k \leqslant p+q\right\} .
$$

Note that $S_{p+q+1}=\mathscr{A}(U)$ and $S_{p+1}=\hat{\mathscr{A}}_{\xi} \subseteq R$. Given $j>p$, assume that $S_{j} \subseteq R$. Define $T_{j}=1-(-1)^{\left|\xi^{j}\right|} \xi^{j}\left(\partial / \partial \xi^{j}\right)$ (no sum). By Theorem 1.1, $\left[T_{j}, \partial / \partial \xi^{k}\right]=0$ for $k \neq j$. $\partial / \partial \xi^{j} \circ T_{j}$ is also 0 . Thus $T_{j}\left(S_{j+1}\right) \subset S_{j}$ and similarly, $\partial / \partial \xi^{j}\left(S_{j+1}\right) \subset S_{j}$. Then given $g \in S_{j+1}, g=T_{j} g+(-1)^{\left|\xi^{j}\right|} \xi^{j}\left(\partial g / \partial \xi^{j}\right) \in R+\xi^{j} R \subseteq R$, so it follows by induction that $S_{j} \subseteq R$ for all $j>p$.

Recall the homeomorphism $\tilde{\xi}: U \rightarrow \tilde{\xi}(U)$ of axiom 2. Given $f \in \mathscr{A}(U), \tilde{f} \circ \tilde{\xi}^{-1}$ is by definition a continuous $B$-valued function on $\tilde{\xi}(U)$.

THEOREM 1.3. For all $f \in \mathscr{A}(U), \tilde{f} \circ \tilde{\xi}^{-1}$ is smooth.

Proof. Let $g=\tilde{f} \circ \tilde{\xi}^{-1}$. Given $x, y \in U$, set $a^{i}=\tilde{\xi}^{i}(x)$ and $b^{i}=\tilde{\xi}^{i}(y)$. Using axiom 3 , set

$$
f=\tilde{f}(x)+f_{i} \cdot\left(\xi^{i}-a^{i}\right) .
$$

Then

$$
\frac{\widetilde{\partial f}}{\partial \xi^{i}}(x)=(-1)^{|f|\left|\xi^{i}\right|} \tilde{f}_{i}(x)
$$

Now set $g_{i}=\tilde{f}_{i} \circ \tilde{\xi}^{-1}$. Then by applying axiom 3 twice, one finds that there are continuous functions $g_{i j}$ such that

$$
g(b)=g(a)+g_{i}(a) \cdot\left(b^{i}-a^{i}\right)+g_{i j}(b)\left(b^{j}-a^{j}\right)\left(b^{i}-a^{i}\right) .
$$


Therefore $g$ is once continuously differentiable, with

$$
d g_{a}\left(c^{1}, \ldots, c^{p+q}\right)=g_{i}(a) c^{i} .
$$

Since $g_{i}(a)=\tilde{f}_{i}(x)$, one finds that

$$
d g_{a}(c)=(-1)^{|f|\left|\xi^{i}\right|} \frac{\widetilde{\partial f}}{\partial \xi^{i}} \circ \tilde{\xi}^{-1}(a) \cdot c^{i} .
$$

Since the argument can be repeated for $\partial f / \partial \xi^{i}$, it follows that $g$ is arbitrarily smooth.

Let $\mathscr{G}$ denote the image of $\mathscr{A}$ under $\delta$. By Theorem $1.3, \mathscr{G}$ endows $M$ with the structure of a smooth manifold modeled on $B^{p, q}$. Note that in (1.6), the differential $d g_{a}$ is the restriction to $B^{p, q}$ of a $B$-linear map on $B^{p / q}$. Thus $\mathscr{G}$ will in general endow $M$ with a structure that is more than smooth. For instance, when $B=B_{L}, \mathscr{G}$ consists of Rogers' $G^{\infty}$ functions. The general properties of $\mathscr{G}$ will be taken up in $\S 2$.

Let $\mathscr{K}$ denote the kernel of $\delta$. The problem now is to characterize $\mathscr{K}$.

Lemma 1.4. Assume that $f$ is independent of $\xi^{p+1}, \ldots, \xi^{p+q}$, i.e., $f \in \hat{\mathscr{A}}_{\xi}$, and that $\tilde{f}=0$. Then $f=0$.

Proof. From the proof of Theorem 1.3, $(-1)^{|f|\left|\xi^{i}\right|} c^{i} \partial \tilde{\partial} / \partial \xi^{i}=0$ for any $c \in B^{p, q}$. If $j \leqslant p$, one can let $c^{i}=\delta^{i j}$, so that in particular $\partial f / \partial \xi^{i}=0$ for $j=1, \ldots, p$. Furthermore, since partial derivatives graded-commute, $\partial f / \partial \xi^{j}$ lies in $\hat{\mathscr{A}}_{\xi}$ for all $j$. Therefore $\widetilde{\partial f} / \partial \xi^{s}=0$ for all sequences $s$. Then by axiom $4, f=0$.

Thus regardless of the choice of $B$, superfunctions that depend only on the even coordinates may always be regarded as functions on the manifold.

In terms of a coordinate system $\xi, \mathscr{K}$ has the following description.

Lemma 1.5. Let $\left.f \in \mathscr{A}\right|_{U}$ and write $f=f_{\mu} \xi^{\mu}$, with $f_{\mu} \in \hat{\mathscr{A}}_{\xi}$ for all $\mu$. Then $\tilde{f}=0$ if and only if for all $\mu, f_{\mu} c=0$ whenever $c$ is a product of $|\mu|$ many odd elements of $B$.

Proof. We may assume $\xi$ is centered at the origin in $B^{p, q}$. For any $\mu$, and any odd elements $c_{1}, \ldots, c_{|\mu|} \in B_{1}$, there is a $q$-tuple $b=\left(b^{1}, \ldots, b^{q}\right) \in B^{0, q}$ such that for all $a \in B^{p, 0}$ with $a$ near 0 , and all $t \in \mathbf{R}$,

$$
\tilde{f}(a, t b)=\tilde{f}_{\mu}(a, 0) t^{|\mu|} c_{1} \cdots c_{|\mu|}+\text { terms of lower order. }
$$

Thus $\tilde{f}=0$ if and only if $\tilde{f}_{\mu}(a, 0) c_{1} \cdots c_{|\mu|}=0$ for all $\mu$ and all $c_{1}, \ldots, c_{|\mu|} \in B_{1}$. Since $\tilde{f}_{\mu}$ is independent of $b^{1}, \ldots, b^{q}$ and since $\delta$ is injective of $\hat{\mathscr{A}}_{\xi}$, the result follows.

It is now possible to characterize $\mathscr{K}$ invariantly.

THEOREM 1.6. $\mathscr{K}$ consists of the ideal $\left\{f \in \mathscr{A} \mid f g_{1} \cdots g_{q}=0\right.$ for all $g_{1}, \ldots, g_{q} \in$ $\left.\mathscr{A}_{1}\right\}$. 
Proof. Assume $\tilde{f}=0$. Let $g_{1}, \ldots, g_{q}$ be odd elements of $\left.\mathscr{A}\right|_{x}$. Let $g=g_{1} \cdots g_{q}$. Choose a coordinate system $\xi$, and let $f=f_{\mu} \xi^{\mu}$ as in Theorem 1.2. Also set $g_{i}=g_{i \nu} \xi^{\nu}$ and set $g=g_{\mu} \xi^{\mu}$. Then

$$
g_{\mu}=\sum_{\nu_{1}+\cdots+v_{q}=\mu} \pm g_{1 \nu_{1}} \cdots g_{q \nu_{q}}
$$

and $\left|g_{i \nu}\right|=1+|\nu|$. It follows that $g_{\mu}$ is a sum of products of at least $(q-|\mu|)$ many odd elements of $\hat{\mathscr{A}}_{\xi}$. Now $f g= \pm f_{\mu} g_{\nu} \xi^{\mu} \xi^{\nu}$. If $|\mu|>q-|\nu|$, then $\xi^{\mu} \xi^{\nu}=0$, and if $|\mu| \leqslant q-|\nu|$, then by Lemma $1.5, f_{\nu} g_{\mu}=0$. Thus $f g=0$.

Conversely, assume $f g=0$ whenever $g$ is a product of $q$-many odd elements of $\left.\mathscr{A}\right|_{x}$. Let $\overrightarrow{1}$ denote the $q$-tuple $(1, \ldots, 1)$. Given $\nu$, let $c_{1}, \ldots, c_{|\nu|}$ be odd elements of $B$. Then $f c_{1} \cdots c_{|\nu|} \xi^{1-\nu}=0$. The top order term of this expression is $\pm f_{\nu} c_{1} \cdots c_{|\nu|} \xi^{\overrightarrow{1}}$. Thus $f_{\nu} c_{1} \cdots c_{|\nu|}=0$ for all $\nu$ and all products $c_{1}, \ldots, c_{\nu}$ of $|\nu|$-many odd elements of $B$. By Lemma $1.5, \tilde{f}=0$.

This section closes with some observations about the derivations of $\mathscr{G}$ and the tangent bundle of $M$.

It is easily shown that every derivation of $\mathscr{G}$ is the image of a $\mathscr{K}$-preserving derivation of $\mathscr{A}$.

\section{Proposition 1.7. The even part of Der $\mathscr{A}$ preserves $\mathscr{K}$.}

Proof. Let $\mathscr{I}_{n} \subset \mathscr{A}$ be the ideal $\left\{f \mid f g_{1} \cdots g_{n}=0\right.$ for all odd $\left.g_{1}, \ldots, g_{n} \in \mathscr{A}\right\}$. Then $\mathscr{I}_{0}=0 \subset \mathscr{I}_{1} \subset \mathscr{I}_{2} \cdots$ and $\mathscr{I}_{q}=\mathscr{K}$ by Theorem 1.6.

Assuming that $\operatorname{Der}_{0} \mathscr{A}$ preserves $\mathscr{I}_{m}$ for all $m<n$, take $Y \in \operatorname{Der}_{0} \mathscr{A}$ and $f \in \mathscr{I}_{n}$. For $g_{1}, \ldots, g_{n} \in \mathscr{A}_{1}$,

$$
g_{1} \cdots g_{n} Y f=g_{1} \cdots g_{n-1} Y\left(g_{n} f\right)-g_{1} \cdots g_{n-1} Y g_{n} \cdot f=g_{1} \cdots g_{n-1} Y\left(g_{n} f\right),
$$

since $Y g_{n}$ is odd. But $g_{n} f \in \mathscr{I}_{n-1}$ since $g_{n}$ is odd. So $g_{1} \cdots g_{n} Y f=0$ by the inductive hypothesis. Then $Y$ preserves $\mathscr{I}_{n}$ for all $n$.

Definition. For $x \in M$, the tangent space to $\mathscr{A}$ at $x$ is the set of $B$-graded-linear maps $v:\left.\mathscr{A}\right|_{x} \rightarrow B$ such that

$$
v(f g)=v f \cdot \tilde{g}(x)+(-1)^{|f||v|} \tilde{f}(x) \cdot v g .
$$

Denote this space by $T_{x} \mathscr{A}$ and set

$$
T \mathscr{A}=\bigcup_{x \in M} T_{x} \mathscr{A}
$$

$T \mathscr{A}$ is called the tangent bundle of $\mathscr{A}$.

Proposition 1.8. For all $x \in M, T_{x} \mathscr{A}$ is a free $(p, q)$-dimensional $B$ module. The even part of $\left(T_{x} \mathscr{A}\right)_{0}$ is canonically isomorphic to the tangent space to $M$ at $x$.

Proof. Fix a coordinate system $\xi$ at $x$. It follows from axiom 3 that $T_{x} \mathscr{A}$ has the basis $\left\{\delta_{x} \circ \partial / \partial \xi^{i}\right\}$, where $\delta_{x}$ is evaluation at $x$.

If $v$ is even, then

$$
v(f)=\left.\frac{d}{d t}\right|_{0} \tilde{f} \circ \tilde{\xi}^{-1}(b+t a),
$$


where $b=\tilde{\xi}(x)$ and $a=\left(v\left(\xi^{1}\right), \ldots, v\left(\xi^{p+q}\right)\right)$. Furthermore, for any $a \in B^{p, q}$ the right-hand side of (1.7) defines a tangent vector to $\mathscr{A}$ at $x$. This identifies $\left(T_{x} \mathscr{A}\right)_{0}$ with the set of all derivatives of smooth curves at $x$.

In summary, on $B^{p, q}$ there is a distinguished sheaf, $\mathscr{G}$, of supersmooth functions, and the structure sheaf of a supermanifold is locally isomorphic to the tensor product of the supersmooth functions of the even coordinates and the Grassmann algebra generated by the odd coordinates. This tensor product does not coincide with $\mathscr{G}$ in general.

$\$ 2$ establishes the consistency of axiomatic definition by verifying the axioms in the local model.

2. The supermanifold over $B^{p, q}$. According to Theorem $1.3, \mathscr{G}$ pulls back to a subsheaf of the sheaf of smooth functions on $B^{p, q}$. The functions obtained in this way satisfy the $G^{\infty}$ condition introduced by Rogers [11] and reviewed below.

Definition 2.1. Let $f: B^{p, 0} \rightarrow B$ be $C^{\infty}$. Say $f$ is $C^{\infty}(B)$, or $B$-smooth, if for all $x \in B^{p, q}, d f_{x}$ is $B_{0}$-linear. Given $g: B^{p, q} \rightarrow B$, say that $g$ is $C^{\infty}(B)$, or $B$-smooth, if $g\left(a^{1}, \ldots, a^{p}, b^{1}, \ldots, b^{q}\right)$ is a polynomial in $b^{1}, \ldots, b^{q}$ whose coefficients are $B$-smooth functions of $\left(a^{1}, \ldots, a^{p}\right) . g$ will be called $C^{\omega}(B)$, or $B$-analytic, if it is given by a power series in $(a, b)$.

Denote the sheaf of germs of $C^{\infty}(B)$ functions on $B^{p, q}$ by $\mathscr{G}^{\infty}$. In [4] it is shown that the $\mathscr{G}^{\infty}$ condition for a function $f$ on $B^{p, q}$ can be described by the condition that $d f$ be $B_{0}$ linear plus an additional set of first and second order conditions. If $B$ is a Banach-Grassmann algebra, then the $B_{0}$-linearity of $d f$ is necessary and sufficient for $f$ to be $C^{\infty}(B)$ [7].

Let $\hat{\mathscr{G}} \subset \mathscr{G}^{\infty}$ denote the subsheaf of germs that are independent of $b^{1}, \ldots, b^{q}$. Define $\hat{\mathscr{G}}^{\omega}$ and $\mathscr{G}^{\omega}$ similarly for $C^{\omega}(B)$ functions. Let $\tilde{\pi}^{i}: B^{p, q} \rightarrow B$ denote the $i$ th projection. Let $\Lambda[q]$ denote the exterior algebra generated by $\tilde{\pi}^{p+1}, \ldots, \tilde{\pi}^{p+q}$. Let $\mathscr{A}^{\infty}=\hat{\mathscr{G}} \otimes \Lambda[q]$. For $i=1, \ldots, p$, let $\pi^{i}=1 \otimes \tilde{\pi}^{i}$ and for $i=p+1, \ldots, p+q$, let $\pi^{i}=\tilde{\pi}^{i} \otimes 1$. From the definition of $\mathscr{G}^{\infty}$ there is a surjection homomorphism

$$
\mathscr{A}^{\infty} \stackrel{\delta^{\infty}}{\rightarrow} \mathscr{G}^{\infty}, \quad f \otimes \pi^{i} \rightarrow f \tilde{\pi}^{i} .
$$

If $(M, \mathscr{A}, \delta)$ is a supermanifold, then by $\S 1$, a coordinate system $\xi$ for $\mathscr{A}$ in a neighborhood $U$ provides an imbedding of paris:

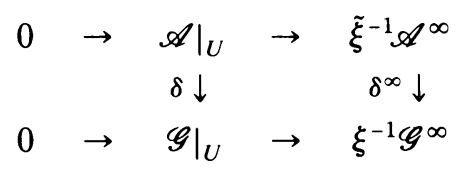

The consistency of the axiomatic definition follows from

TheOREM 2.2. The triples $\left(B^{p, q}, \mathscr{A}^{\infty}, \delta^{\infty}\right)$ and $\left(B^{p, q}, \mathscr{A}^{\omega}, \delta^{\omega}\right)$ are supermanifolds.

In light of Theorem 2.2, a supermanifold $(M, \mathscr{A}, \delta)$ will be called $C^{\omega}(B)$ or $B$-analytic if every coordinate system in $\mathscr{A}$ maps $\mathscr{G}$ to $\mathscr{G}^{\omega}$. Otherwise $(M, \mathscr{A}, \delta)$ will be called $C^{\infty}(B)$ or $B$-smooth. 
Theorem 2.2 will be proved at the end of this section, after some preliminary results.

LeMma 2.3. Let $x \in B^{p, q}$, and let $U$ be a star-shaped neighborhood of $x$. Then for all $f \in \mathscr{G}^{\infty}(U)$ there exist $g_{1}, \ldots, g_{p+q} \in \mathscr{G}^{\infty}(U)$ such that

$$
f=f(x)+g_{i}\left(\tilde{\pi}^{i}-\tilde{\pi}^{i}(x)\right) .
$$

Proof. This is a straightforward generalization of the proof in the $C^{\infty}$ case. Let $d f(c)=f_{i} c^{i}$ for some $C^{\infty}(B)$ functions $f_{1}, \ldots, f_{p+q}$. Then for $y \in U$,

$$
\begin{aligned}
f(y) & =f(x)+\int_{0}^{1} \frac{d}{d t} f((1-t) x+t y) d t \\
& =f(x)+g_{i}(y)\left(\tilde{\pi}^{i}(y)-\tilde{\pi}^{i}(x)\right),
\end{aligned}
$$

where

$$
g_{i}(y)=\int_{0}^{1} f_{i}((1-t) x+t y) d t .
$$

Since $f_{i}$ is $C^{\infty}(B)$, it follows that $g_{i}$ is $C^{\infty}(B)$ as well.

Let $f \in \hat{\mathscr{G}}$. For $i=1, \ldots, p$, define

$$
\frac{\partial}{\partial \pi^{i}} f=\left(d f, e_{i}\right)
$$

where $e_{i}$ is the vector whose $j$ th entry is $\delta_{i}^{j}$. Extend $\partial / \partial \pi^{i}$ to $\mathscr{A}^{\infty} \Lambda[q]$-linearly. For $k=p+1, \ldots, p+q$, let $\partial / \partial \pi^{k}: \Lambda[q] \rightarrow \Lambda[q]$ be the derivation generated by

Extend $\partial / \partial \pi^{k}$ to $\mathscr{A}^{\infty} \hat{G}$-linearly.

$$
\frac{\partial}{\partial \pi^{k}}\left(\pi^{j}\right)=(-1)^{\left|\pi^{j}\right|\left|\pi^{k}\right|} \delta_{k}^{j}
$$

LemMa 2.4. Der $\mathscr{A}^{\infty}$ is freely generated over $\mathscr{A}^{\infty}$ by $\partial / \partial \pi^{1}, \ldots, \partial / \partial \pi^{p+q}$.

Proof. This is straightforward.

Again for $F \in \mathscr{A}^{\infty}$, denote $\delta^{\infty}(F)$ by $\tilde{F}$. Then for $c \in B^{p, q}$, it can be verified that

$$
d \tilde{F}\left(c^{1}, \ldots, c^{p+q}\right)=(-1)^{|F|\left|\pi^{i}\right|} \frac{\widetilde{\partial F}}{\partial \pi^{i}} c^{i} .
$$

LemMa 2.5. (1) Let $f: B^{p, q} \rightarrow B^{r, s}$ and $g: B^{r, s} \rightarrow B$ be $C^{\infty}(B)$ functions. Then $g \circ f$ is also $C^{\infty}(B)$.

(2) Let $\varphi: B^{p, q} \rightarrow B^{p, q}$ be a $C^{\infty}(B)$ function which is also a diffeomorphism. Then $\varphi^{-1}$ is also $C^{\infty}(B)$.

Proof. (1) Let $f=\tilde{F}, g=\tilde{G}$. Then for $c \in B^{p, q}$,

$$
d(f \circ g)\left(c^{1}, \ldots, c^{p+q}\right)=\overbrace{d F^{k}\left(\frac{\partial}{\partial \pi^{i}}\right)} \overbrace{d G\left(\frac{\partial}{\partial \pi^{k}}\right)} c^{i}
$$

and hence is of the form $q_{i} c^{i}$ for some $C^{\infty}(B)$ functions $q_{i}$. Thus $f \circ g$ is $C^{\infty}(B)$.

(2) is proved similarly.

By Lemma 2.5, there is a category of $C^{\infty}(B)$ manifolds. 
LEMMA 2.6. Let $U$ be a neighborhood in $B^{p, q}$ and let $\xi^{1}, \ldots, \sigma x^{p+q} \in \mathscr{A}^{\infty}(U)$ be a coordinate system. Then $\left(\tilde{\xi}^{1}, \ldots, \widehat{\xi^{p+q}}\right)$ is a diffeomorphism from $U$ to a neighborhood $V \subset B^{p, q}$.

Proof. Let $\tilde{\xi}=\left(\tilde{\xi}^{1}, \ldots, \tilde{\xi}^{p+q}\right)$. Let $c=\left(c^{1}, \ldots, c^{p+q}\right) \in B^{p, q}$. Then by 2.1 ,

$$
d \tilde{\xi}(c)=(-1)^{\left|\xi^{j}\right|\left|\pi^{i}\right|} \frac{\partial \xi^{j}}{\partial \pi^{i}} c^{i} .
$$

Since $\xi$ is a coordinate system, $d \xi^{j}\left(\partial / \partial \pi^{i}\right)$ is an invertible matrix, so $\delta$ of it is invertible as well. Then $\tilde{\xi}$ is a diffeomorphism by the inverse function theorem.

The next theorem establishes the equivalence of coordinate systems and isomorphisms of $\mathscr{A}^{\infty}$.

THEOREM 2.7. Let $U$ be a neighborhood in $B^{p, q}$ and let $\xi=\left(\xi^{1}, \ldots, \xi^{p+q}\right) \in \mathscr{A}^{\infty}(U)$ form a coordinate system. Let $V=\tilde{\xi}(U)$. Then there is a unique sheaf isomorphism $\hat{\xi}$ satisfying

$$
\begin{array}{ccc}
\tilde{\xi}^{-1}\left(\left.\mathscr{A}^{\infty}\right|_{V}\right) & \stackrel{\hat{\xi}}{\rightarrow} & \left.\mathscr{A}^{\infty}\right|_{U} \\
\downarrow \delta^{\infty} & & \downarrow \delta^{\infty} \\
\tilde{\xi}^{-1}\left(\left.\mathscr{G}^{\infty}\right|_{V}\right) & \stackrel{\tilde{\xi}^{*}}{\rightarrow} & \left.\mathscr{G}^{\infty}\right|_{U}
\end{array}
$$

such that

$$
\hat{\xi}\left(\left.\pi^{i}\right|_{V}\right)=\xi^{i} \text { for } i=1, \ldots, p+q
$$

Proof. The easiest proof, which is sketched here, foreshadows the functor of points, which is reviewed in $\$ 5$.

Let $n \geqslant q$. For each $x \in V$, let $\sigma^{-1}(x)$ be the set of graded $B$-algebra homomorphisms $h$ from $\left.\mathscr{A}^{\infty}\right|_{x}$ to $B \otimes \Lambda[n]$ such that

$$
\begin{array}{ccc}
\left.\mathscr{A}^{\infty}\right|_{x} & \stackrel{h}{\rightarrow} & B \otimes \Lambda[n] \\
\delta^{\infty} \downarrow & & \downarrow \varepsilon \\
\left.\mathscr{G}^{\infty}\right|_{x} & \stackrel{\delta_{x}}{\rightarrow} & B
\end{array}
$$

commutes. $\delta_{x}$ is evaluation at $x$ and $B \otimes \Lambda[n] \stackrel{\varepsilon}{\rightarrow} B$ is the $B$-linear homomorphism induced by the R-linear homomorphism $\Lambda[n] \rightarrow \mathbf{R}$. Let

$$
\bar{V}=\bigcup_{x \in V} \sigma^{-1}(x) \text {. }
$$

In $\S 5$ it is shown that $\bar{V}$ can be identified with $\varepsilon^{-1}(V)$, where $(B \otimes \Lambda[n])^{p, q} \stackrel{\varepsilon}{\rightarrow} B^{p, q}$ is $\varepsilon$ in each component.

For $f \in \mathscr{A}^{\infty}(V)$, one defines $\bar{f}: \bar{V} \rightarrow B \otimes \Lambda[n]$ by $\bar{f}(h)=h(f)$. Let $T(f)=\bar{f}$. Then it happens that $f \stackrel{T}{\rightarrow} \bar{f}$ is $1-1$ for $n \geqslant q$, and $\bar{f}$ is a $C^{\infty}(B \otimes \Lambda[n])$ function on $\bar{V}$. (See §5.) 
Next, let $\bar{\xi}=\left(\bar{\xi}^{1}, \ldots, \overline{\xi^{p+q}}\right) \cdot \bar{\xi}$ defines a $C^{\infty}(B \otimes \Lambda[n])$ diffeomorphism from $\bar{U}$ to $\bar{V}$. The map $x \rightarrow \delta_{x}$ is an imbedding of $U$ in $\bar{U}$. Then the isomorphism $\hat{\xi}$ is given by pullback: $f \rightarrow T^{-1}(\bar{f} \circ \bar{\xi})$.

THEOREM 2.2. The triples $\left(B^{p, q}, \mathscr{A}^{\infty}, \delta^{\infty}\right)$ and $\left(B^{p, q}, \mathscr{A}^{\omega}, \delta^{\omega}\right)$ are supermanifolds.

Proof. It remains to verify axioms 3 and 4 .

Axiom 3 is the Taylor expansion. In the coordinate system $\pi^{1}, \ldots, \pi^{p+q}$ this is obvious. Given another coordinate system $\xi^{1}, \ldots, \xi^{p+q}$, form the isomorphism $\hat{\xi}$ of Theorem 2.6. for $x \in B^{p, q}$ and $\left.f \in \mathscr{A}^{\infty}\right|_{x}$, let $\hat{\xi}^{-1} f=\tilde{f}(x)+f_{i} \cdot\left(\pi^{i}-\tilde{\xi}^{i}(x)\right)$. Then $f=\tilde{f}(x)+\hat{\xi} f_{i} \cdot\left(\xi^{i}-\tilde{\xi}^{i}(x)\right)$, as desired.

For axiom 4, take $f_{\mu} \otimes \pi^{\mu} \in \mathscr{A}^{\infty}$ and assume $X_{1} \cdots X_{n}\left(f_{\mu} \otimes \pi^{\mu}\right) \in \operatorname{ker} \delta^{\infty}$, for all derivations $X_{1}, \ldots, X_{n}$. Then each $f_{\mu}$ is in $\operatorname{ker} \delta^{\infty}$. Since $\delta^{\infty}$ is $1-1$ on $\hat{\mathscr{G}}$ by construction, it follows that $f_{\mu} \otimes \pi^{\mu}=0$.

By combining $\S 1$ and Theorem 2.2 one arrives at the following:

Structural definition of $a C^{\infty}(B)$ supermanifold. Given a $C^{\infty}(B)$ manifold $M$ with structure sheaf $\mathscr{G}$, and a coordinate system $\xi=\left(\xi^{1}, \ldots, \xi^{p+q}\right)$ on a neighborhood $U \subset M$, let

$$
\hat{\mathscr{A}}_{\xi}=\left\{\left.f \in \mathscr{G}^{\infty}\right|_{U}:\left.f \circ \xi^{-1} \in \hat{\mathscr{G}}\right|_{\xi(U)}\right\} .
$$

Then define $\left.\hat{\mathscr{A}}_{\xi} \otimes \Lambda[q] \stackrel{\delta_{\xi}}{\rightarrow} \mathscr{G}\right|_{U}$ by $f \otimes \pi^{\mu} \rightarrow f \cdot \xi^{\mu}$. Then a $C^{\infty}(B)$ supermanifold is a triple $(M, \mathscr{A}, \delta)$, where $\mathscr{A}$ is a sheaf of $\mathbf{Z}_{2}$-graded-commutative algebras and $\delta$ is a surjective morphism $\mathscr{A} \stackrel{\delta}{\rightarrow} \mathscr{G}$, with the property that if for any $x \in M$ there is a $C^{\infty}(B)$ coordinate system $\xi$ in a neighborhood $U$ of $x$, and an isomorphism $\hat{\xi}:\left.\mathscr{A}\right|_{U} \rightarrow \hat{\mathscr{A}}_{\xi} \otimes \Lambda[q]$ such that

$$
\begin{gathered}
\left.\mathscr{A}\right|_{U} \stackrel{\hat{\xi}}{\rightarrow} \hat{\mathscr{A}}_{\xi} \otimes \Lambda[q] \\
\delta \searrow \mathscr{G}_{U} \prec \delta_{\xi}
\end{gathered}
$$

commutes.

$C^{\omega}(B)$ supermanifolds are defined analogously.

3. Supermanifolds over a commutative ground ring. Assume now that $B$ is commutative. Then the axioms of $\$ 1$ yield what is essentially the supermanifold theory of Berezin and Kostant, with mild generalization owing to the arbitrariness of $B$. The exposition here is brief, and the situation is not much different from the special case $B=C$, which is treated in [12].

Lemma 3.1. Let $(M, \mathscr{A}, \delta)$ be a $C^{\infty}(B)$ supermanifold.

(1) $\operatorname{ker} \delta$ is the ideal generated by $\mathscr{A}_{1}$.

(2) $\mathscr{A}$ is locally isomorphic to $\mathscr{G} \otimes \Lambda[q]$. 
Proof. Locally one may write $\left.\mathscr{A}\right|_{U}=\hat{\mathscr{A}}_{\xi} \otimes \Lambda[q]$. Since $B$ has no odd part, $\delta_{\xi}\left(\pi^{i}\right)=0$ for $i \geqslant p$. Moreover, $\delta_{\xi}$ is injective on $\hat{\mathscr{A}}_{\xi}$. So both claims follow.

Set $\mathscr{E}=\mathscr{A}_{1} / \mathscr{A}_{1}^{2}$. Then $\mathscr{E}$ is a $\mathscr{G}$-module, and by Lemma 3.1, $\mathscr{E}$ is locally free. Let $\Lambda \mathscr{E}$ denote the presheaf $U \rightarrow \Lambda(\mathscr{E}(U))$, where $\Lambda(\mathscr{E}(U))$ is the exterior algebra of $\mathscr{E}(U)$ over $\mathscr{G}(U)$. For each $x \in U$, there is a natural map $\Lambda(\mathscr{E}(U)) \stackrel{\alpha}{\rightarrow} \Lambda\left(\left.\mathscr{E}\right|_{x}\right)$. Conversely, the direct limit of the diagrams

$$
\begin{array}{ccc}
\mathscr{E}(U) . & \rightarrow & \Lambda(\mathscr{E}(U)) \\
\downarrow & & \downarrow \\
\mathscr{E}(V) & \rightarrow & \Lambda(\mathscr{E}(V)), \quad V \subset U,
\end{array}
$$

gives a map $\left.\mathscr{E}_{x} \rightarrow \Lambda \mathscr{E}\right|_{x}$ from which $\left.\Lambda\left(\mathscr{E}_{x}\right) \stackrel{\beta}{\rightarrow}(\Lambda \mathrm{E})\right|_{x}$ follows. Then $\alpha=\beta^{-1}$, so $\left.(\Lambda \mathscr{E})\right|_{x}=\Lambda\left(\left.\mathscr{E}\right|_{x}\right)$

$\Lambda \mathscr{E}$ is $\mathbf{Z}$-graded. Denote the $i$ th piece of $\Lambda \mathscr{E}$ by $\Lambda^{i} \mathscr{E}$, and let $\Lambda^{(j)} \mathscr{E}=\sum_{i \geqslant j} \Lambda^{i} \mathscr{E}$. Then $\Lambda^{0} \mathscr{E}=\mathscr{G}$, and the map $0 \rightarrow \Lambda^{(1)} \mathscr{E} \rightarrow \Lambda \mathscr{E} \rightarrow \mathscr{G} \rightarrow 0$ makes $\Lambda \mathscr{E}$ a supermanifold.

Given a $\mathbf{Z}_{2}$-graded $B$-linear algebra automorphism $g$ of $\Lambda \mathscr{E}, g$ induces an automorphism $\mathscr{G} \stackrel{g_{0}}{\rightarrow} \mathscr{G}$ by virtue of the identification $\mathscr{G} \approx \Lambda \mathscr{E} / \Lambda^{(1)} \mathscr{E} . g_{0}$ need not be the identity. For example, if $M=B^{p, q}$, and $x$ is a point in $B^{p, q}$ each of whose coordinates is nilpotent, then

$$
g_{x}: \mathscr{G} \rightarrow \mathscr{G}, \quad g_{x}(f)(y)=f(y+x)
$$

is a nontrivial automorphism. More generally, if $X$ is a global section of $(\operatorname{Der} \mathscr{G})_{0}$ and $X$ is nilpotent, then $\exp X$ defines an automorphism of $\mathscr{G}$. Thus, in contrast to the special case of real or complex manifolds, in which the structure sheaf has no nontrivial automorphisms, the group of automorphisms of $\mathscr{G}$ is related to the geometry of $M$.

[12] describes deformations of complex supermanifolds. The results there carry over to this more general setting. In particular, to every supermanifold $(M, \mathscr{A}, \delta)$ over a commutative ground ring $B$, one may associate a class $\Delta \mathscr{A} \in$ $H^{1}(M, \operatorname{Der} \Lambda \mathscr{E}) / G$, where $G$ is a subgroup of $H^{0}(M$, Aut $\Lambda \mathscr{E})$, such that $\Delta \mathscr{A}$ vanishes if and only if $\mathscr{A}$ and $\Lambda \mathscr{E}$ are isomorphic. Further, under suitable conditions one can produce a supermanifold $\mathscr{A}$ with a prescribed value for $\Delta \mathscr{A}$. If $B=\mathbf{R}$, then $\mathscr{G}$ reduces to the sheaf of $C^{\infty}$ functions on $M$. In that case $\Delta \mathscr{A}$ is necessarily 0 . This yields the theorem first proved by Gawedzki [6] and Batchelor [1], which states that every $C^{\infty}$ supermanifold is $\Lambda \mathscr{E}$ for some locally free sheaf $\mathscr{E}$.

4. $B=\Lambda[L]$. Consider now the case developed first by Rogers, in which $B$ is a finite-dimensional exterior algebra.

Let $F=\mathbf{R}$ or $\mathbf{C}$. Fix an integer $L$ and let $B=\Lambda\left(F^{L}\right)$.

It turns out that if $(M, \mathscr{A}, \delta)$ is a $C^{\infty}(B)$ supermanifold, then $\delta$ is not injective. This is a consequence of the fact that there is an ambiguity in passing from a polynomial function on $B^{0, q}$ to an element of $B \otimes \Lambda[q]$. This ambiguity diminishes as $L$ tends to $\infty$, and one might hope that for large values of $L, \mathscr{A}$ is determined by $\mathscr{G}$. However, this is not the case, as is shown in $\$ 5$, nor is it known whether an arbitrary $C^{\infty}(B)$ manifold $(M, \mathscr{G})$ with $0<L<\infty$ always admits a supermanifold 
extension. $L=q$ is a critical value, for if $L \geqslant q$ one finds that the kernel of $\delta$ is intrinsic to $\mathscr{G}$. Then the problem of obtaining $(\mathscr{A}, \delta)$ is that of filling in the middle term of the sequence $0 \rightarrow \mathscr{K} \rightarrow \mathscr{A} \rightarrow \mathscr{G} \rightarrow 0$. The obstruction to the existence of this sequence lies in $H^{2}(M, \operatorname{Der}(\mathscr{G}, \mathscr{K}))$, and if the obstruction vanishes, the supermanifolds over $M$ are classified by $H^{1}(M, \operatorname{Der}(\mathscr{G}, \mathscr{K}))$.

Let $J \subset B$ denote the nilpotent ideal, so that $B=F+J$. Then $B^{p, q}=F^{p} \dot{+} J^{p, q}$. De Witt refers to this decomposition as "body" and "soul" [5]. Let $\varepsilon$ denote the projection $B^{p, q} \stackrel{\varepsilon}{\rightarrow} F^{p}$. Let $\mathscr{C}$ denote the sheaf of smooth $(F=\mathbf{R})$ or holomorphic $(F=\mathbf{C})$ functions on $F^{p}$. Given $x \in F^{p}$ and $\left.f \in \mathscr{C}\right|_{x} \otimes B, f$ extends by Taylor expansion to a section of $\hat{G}$ along $\varepsilon^{-1}(x)$. Thus the Taylor expansion defines an isomorphism $\hat{\mathscr{G}} \approx \varepsilon^{-1}(\mathscr{C} \otimes B)$, and in turn one has the isomorphism

$$
\mathscr{A}^{\infty} \approx \varepsilon^{-1}(\mathscr{C} \otimes B \otimes \Lambda[q]) \text {. }
$$

LEMMA 4.1. Let $(M, \mathscr{A}, \delta)$ be a $(p, q)$-dimensional $C^{\infty}(B)$ supermanifold. Let I denote the ideal of nilpotents in $\mathscr{A}$. Then $\operatorname{ker} \delta=I^{L+1}$.

Proof. One may assume $M=B^{p, q}$ and $\mathscr{A}=\hat{\mathscr{G}} \otimes \Lambda[q]$. Take $x \in B^{p, q}$ and let $\left.f \in \mathscr{A}\right|_{x}$. Write $x=y+n$, with $y \in F^{p}$ and $n \in J^{p, q}$. Let $\beta^{1}, \ldots, \beta^{L}$ be generators for $\Lambda\left(F^{L}\right)$. Then $f=\varepsilon^{-1}\left(f_{\mu \nu} \otimes \beta^{\mu} \otimes \pi^{\nu}\right)$, for some $\left.f_{\mu \nu} \in \mathscr{C}\right|_{\varepsilon(x)} . f$ lies in ker $\delta$ if and only if $b^{\nu} \beta^{\mu} f_{\mu \nu}(y)=0$ for all $b^{1}, \ldots, b^{q} \in B_{1}$ and all $y \in F^{p}$ sufficiently close to $\varepsilon(x)$. This holds if and only if $f_{\mu \nu}$ vanishes near $\varepsilon(x)$ whenever $|\mu|+|\nu| \leqslant L$. Since $\mathscr{I}$ is generated by $\pi^{1}, \ldots, \pi^{q}, \beta^{1}, \ldots, \beta^{L}$, the result follows.

Henceforth assume $L \geqslant q$.

THEOREM 4.2. $\operatorname{ker} \delta$ is a $\mathscr{G}$ module, isomorphic to the $(L+1)$ st symmetric power of $\mathscr{N}$ over $\mathscr{G}$.

Proof. Let $\mathscr{K}$ denote the $(L+1)$ st symmetric power of $\mathscr{N}$ over $\mathscr{G}$. That is, $\mathscr{K}$ is the $(L+1)$ st tensor power of $\mathscr{N}$ over $\mathscr{G}$, modulo the relations

$$
f_{1} \otimes \cdots \otimes f_{i} \otimes f_{i+1} \otimes \cdots \otimes f_{n}-(-1)^{\left|f_{i}\right|\left|f_{i+1}\right|} f_{1} \otimes \cdots \otimes f_{i+1} \otimes f_{i} \otimes \cdots \otimes f_{n} .
$$

By Lemma 4.1, $(\operatorname{ker} \delta)^{2}=\mathscr{I}^{2 L+2}=0$, since $2 L+2 \geqslant L+q+1$. Thus $\operatorname{ker} \delta$ is a $\mathscr{G}$-module. In fact, $\mathscr{I}^{2 L+1}$ is 0 , so the map

$$
\stackrel{\mathscr{K}}{\rightarrow} \mathscr{I}^{L+1}, \quad \tilde{f}_{1} \otimes \cdots \otimes \tilde{f}_{L+1} \rightarrow f_{1} \cdots f_{L+1}
$$

is a well-defined map of $\mathscr{G}$-modules. That $T$ is surjective follows from the definition of $\mathscr{I}^{L+1}$ and the fact that $\mathscr{N}=\delta(\mathscr{I})$. To construct a left inverse for $T$, take $f \in \mathscr{I}^{L+1}$. Assuming, as one may, that $M=B^{p, q}$, write

$$
f=\sum_{|\mu|+|\nu| \geqslant L+1} \varepsilon^{-1}\left(\pi^{\mu} \otimes \beta^{\nu} \otimes f_{\mu \nu}\right), \quad f_{\mu \nu} \in \mathscr{C} .
$$

For each pair $(\mu, \nu)$, partition $\pi^{\mu} \otimes \beta^{\nu}$ into a product $a_{1}^{\mu \nu} \cdots a_{L+1}^{\mu \nu}$ of nilpotent elements. Then $f \rightarrow a_{1}^{\mu \nu} \otimes \cdots \otimes a_{L+1}^{\mu \nu} f_{\mu \nu}$ is a left inverse to $T$, provided it is well defined. If $b_{1}^{\mu \nu}, \ldots, b_{L+1}^{\mu \nu}$ is a different decomposition of $\pi^{\mu} \otimes \beta^{\nu}$, then one can pass from $a_{1}^{\mu \nu} \otimes \cdots \otimes a_{L+1}^{\mu \nu}$ to $b_{1}^{\mu \nu} \otimes \cdots \otimes b_{L+1}^{\mu \nu}$ by moving $\pi$ 's and $\beta$ 's past the $\otimes$ sign, so the map is indeed well defined. 
174

M. J. ROTHSTEIN

The obstruction to the existence of an extension $0 \rightarrow \mathscr{K} \rightarrow \mathscr{A} \rightarrow \mathscr{G} \rightarrow 0$ is a class $c[M] \in H^{2}(M, \operatorname{Der}(\mathscr{G}, \mathscr{K}))$ which can be described as follows.

Cover $M$ by $C^{\infty}(B)$ coordinate systems $\left(U_{\alpha}, \xi \alpha\right)$. Let

$$
\hat{\mathscr{G}}_{\alpha}=\left\{f \in \mathscr{G}_{U_{\alpha}}: f \text { is independent of } \xi_{\alpha}^{p+1}, \ldots, \xi_{\alpha}^{p+q}\right\} .
$$

Let $\mathscr{A}_{\alpha}=\hat{\mathscr{G}}_{\alpha} \otimes \Lambda[q]$ and define

$$
\left.\mathscr{A}_{\alpha} \stackrel{\delta_{\alpha}}{\rightarrow} \mathscr{G}\right|_{U_{\alpha}}, \quad f \otimes \pi^{i} \rightarrow f \cdot \xi_{\alpha}^{i} .
$$

Then $\left(U_{\alpha}, \mathscr{A}_{\alpha}, \delta_{\alpha}\right)$ is a supermanifold.

LEMMA 4.3. For all $\alpha$ and $\beta$ there is an isomorphism $\left.\left.\mathscr{A}_{\alpha}\right|_{U_{\alpha} \cap U_{\beta}} \stackrel{\hat{\xi}_{\beta \alpha}}{\rightarrow} \mathscr{A}_{\beta}\right|_{U_{\alpha} \cap U_{\beta}}$ and an exact sequence $\left.\left.0 \rightarrow \mathscr{K}\right|_{U_{\alpha}} \stackrel{\iota_{\alpha}}{\rightarrow} \mathscr{A}_{\alpha} \stackrel{\delta_{\alpha}}{\rightarrow} \mathscr{G}\right|_{U_{\alpha}} \rightarrow 0$ such that

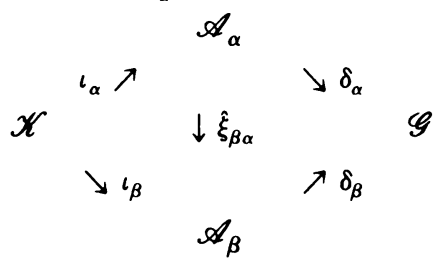

commutes.

Proof. Let $\xi_{\alpha}$ denote the coordinate system $\left(\xi_{\alpha}^{1}, \ldots, \xi_{\alpha}^{p}, \pi^{p+1}, \ldots, \pi^{p+q}\right)$ for $\mathscr{A}_{\alpha}$. For $i=1, \ldots, p+q$, choose sections $\xi_{\alpha \beta}^{i} \in \mathscr{A}_{\alpha}\left(U_{\alpha} \cap U_{\beta}\right)$ such that $\delta_{\alpha}\left(\xi_{\alpha \beta}^{i}\right)=\xi_{\beta}^{i}$. Since $\xi_{\alpha \beta}$ projects onto the coordinate system $\xi_{\beta}$, and since $\operatorname{ker} \delta_{\alpha}$ is nilpotent, $\dot{\xi}_{\alpha \beta}$ is a coordinate system for $\left.\mathscr{A}_{\alpha}\right|_{v_{\alpha} \cap U_{\beta}}$.

Form the isomorphism

$$
\begin{array}{ccc}
\mathscr{A}_{\alpha} & \stackrel{\xi_{\alpha}}{\rightarrow} & \xi_{\alpha}^{-1}\left(\left.\hat{\mathscr{G}}\right|_{\xi_{\alpha}\left(U_{\alpha}\right)} \otimes \Lambda[q]\right) \\
\downarrow \delta_{\alpha} & & \downarrow \delta^{\infty} \\
\left.\mathscr{G}\right|_{U_{\alpha}} & \stackrel{\left(\xi^{-1}\right)^{*}}{\rightarrow} & \xi_{\alpha}^{-1}\left(\left.\mathscr{G}^{\infty}\right|_{\xi_{\alpha}\left(U_{\alpha}\right)}\right), \\
& \hat{\xi}_{\alpha}\left(f_{\mu} \otimes \pi^{\mu}\right)=f_{\mu} \circ \xi_{\alpha}^{-1} \otimes \pi^{\mu} .
\end{array}
$$

Then $\delta^{\infty} \circ \hat{\xi}_{\alpha}\left(\xi_{\alpha \beta}\right)=\left(\xi_{\alpha}^{-1}\right)^{*} \circ \delta_{\alpha}\left(\xi_{\alpha \beta}\right)=\xi_{\beta} \circ \xi_{\alpha}^{-1}$. By Theorem 2.7 there is an isomerphis

$$
\begin{array}{ccc}
\xi_{\alpha}^{-1}\left(\left.\hat{\mathscr{G}} \otimes \Lambda[q]\right|_{\xi_{\alpha}\left(U_{\alpha} \cap U_{\beta}\right)}\right) & \stackrel{\tau}{\rightarrow} & \xi_{\beta}^{-1}\left(\left.\hat{\mathscr{G}} \otimes \Lambda[q]\right|_{\xi_{\beta}\left(U_{\alpha} \cap U_{\beta}\right)}\right) \\
\delta_{\alpha} \downarrow & & \delta^{\infty} \downarrow \\
\xi_{\alpha}^{-1}\left(\left.\mathscr{G}^{\infty}\right|_{\xi_{\alpha}\left(U_{\alpha} \cap U_{\beta}\right)}\right) & \stackrel{\left(\xi_{\alpha}^{\circ} \xi_{\beta}^{-1}\right)^{*}}{\rightarrow} & \xi_{\beta}^{-1}\left(\mathscr{G}_{\xi_{\beta}\left(U_{\alpha} \cap U_{\beta}\right)}\right)
\end{array}
$$

such that $\tau\left(\pi^{i}\right)=\hat{\xi}_{\alpha}\left(\dot{\xi}_{\alpha \beta}^{i}\right)$. Set $\hat{\xi}_{\beta \alpha}=\hat{\xi}_{\beta}^{-1} \circ \tau \circ \hat{\xi}_{\alpha}$. Then $\hat{\xi}_{\beta \alpha}$ satisfies:

$$
\begin{array}{cl}
\mathscr{A}_{\alpha} & \stackrel{\xi_{\beta \alpha}}{\rightarrow} \mathscr{A}_{\beta} \\
\delta_{\alpha} \searrow & \swarrow \delta_{\beta}
\end{array}
$$


Now let $\mathscr{I}_{\alpha}$ be the nilpotent ideal in $\mathscr{A}_{\alpha}$. By Theorem 4.3 and its proof, there is an isomorphism $\left.\mathscr{I}_{\alpha}^{L+1} \stackrel{\rho_{\alpha}}{\rightarrow} \mathscr{K}\right|_{U_{\alpha}}$ characterized by the property that for $f_{1}, \ldots, f_{L+1} \in \mathscr{I}_{\alpha}$,

$$
\rho_{\alpha}\left(f_{1} \cdots f_{L+1}\right)=\delta_{\alpha}\left(f_{1}\right) \otimes \cdots \otimes \delta_{\alpha}\left(f_{L+1}\right) .
$$

Let $i_{\alpha}=\rho_{\alpha}^{-1}$. It remains to show that

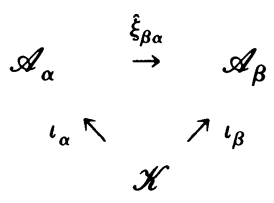

commutes. It is sufficient to check this on monomials

$$
\xi_{\alpha}^{\mu_{1}} \otimes \cdots \otimes \xi_{\alpha}^{\mu_{s}} \otimes b^{1} \otimes \cdots \otimes b^{L+1-s}, \quad b^{i} \in B_{1},
$$

since these generate $\mathscr{K}$ over $\mathscr{G}$.

$$
\begin{aligned}
\rho_{\beta} \circ \hat{\xi}_{\beta \alpha} \circ \iota_{\alpha} & \left(\xi_{\alpha}^{\mu_{1}} \otimes \cdots \otimes \xi_{\alpha}^{\mu_{s}} \otimes b^{1} \otimes \cdots \otimes b^{L+1-s}\right) \\
& =\rho_{\beta} \circ \hat{\xi}_{\beta \alpha}\left(\xi_{\alpha}^{\mu_{1}} \cdots \xi_{\alpha}^{\mu_{s}} \otimes b^{1} \otimes \cdots \otimes b^{L+1-s} L\right) \\
& =\rho_{\beta}\left(\xi_{\beta \alpha}^{\mu_{1}} \cdots \xi_{\beta \alpha}^{\mu_{s}} \cdot b^{1} \cdots b^{L+1}\right) \\
& =\delta_{\beta}\left(\xi_{\beta \alpha}^{\mu_{1}}\right) \otimes \cdots \otimes \delta_{\beta}\left(\xi_{\beta \alpha}^{\mu_{s}}\right) \otimes b^{1} \otimes \cdots \otimes b^{L+1-s} \\
& =\xi_{\alpha}^{\mu_{1}} \otimes \cdots \otimes \xi_{\alpha}^{\mu_{s}} \otimes b^{1} \otimes \cdots \otimes b^{L+1-s}
\end{aligned}
$$

So $\rho_{\beta} \circ \hat{\xi}_{\beta \alpha} \circ \iota_{\alpha}=\mathrm{id}$, as desired.

Consider the collection of diagrams:

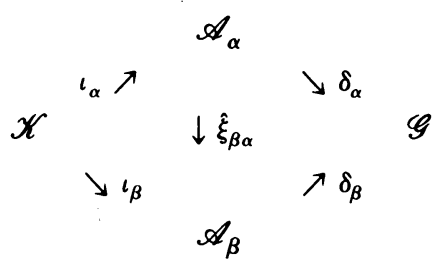

Set $\xi_{\alpha \beta \gamma}=\hat{\xi}_{\alpha \beta} \hat{\xi}_{\beta \gamma} \hat{\xi}_{\gamma \alpha}$. Then $\xi_{\alpha \beta \gamma}(f)=f+\psi_{\alpha \beta \gamma}(f)$, for some $\psi_{\alpha \beta \gamma} f \in \mathscr{I}_{\alpha}^{L+1}$. Since $\left(\mathscr{I}_{\alpha}^{L+1}\right)^{2}=0$,

$$
\xi_{\alpha \beta \gamma}(f g)=f g+f \cdot \psi_{\alpha \beta \gamma}(g)+g \psi_{\alpha \beta \gamma}(f) .
$$

From the diagrams $(\xi), \psi_{\alpha \beta \gamma}$ is of the form $\iota_{\alpha} \circ \Delta_{\alpha \beta \gamma}(\xi) \circ \delta_{\alpha}$ for some $\Delta_{\alpha \beta \gamma}(\xi) \in$ $\operatorname{Der}(\mathscr{G}, \mathscr{K})\left(U_{\alpha} \cap U_{\beta} \cup U_{\gamma}\right)$.

LEMMA 4.4. $\Delta(\xi)$ is a Čech cocycle.

PROOF. Fix four indices, say 1, 2, 3, 4. Restrict $\mathscr{A}_{\alpha}$ and $\hat{\xi}_{\alpha \beta}$ to $U_{1} \cap U_{2} \cap U_{3} \cap U_{4}$. Define $\tau_{\alpha}=\hat{\xi}_{\alpha 1}$ on this intersection. Then the maps $\tau_{\alpha} \tau_{\beta}^{-1}$ also satisfy diagrams $(\xi)$. Thus $\tau_{\alpha} \tau_{\beta}^{-1}=\hat{\xi}_{\alpha \beta}+\iota_{\alpha}^{\circ} \circ \varepsilon_{\alpha \beta} \circ \delta_{\beta}$ for some $\varepsilon_{\alpha \beta}$ belonging to

$$
\operatorname{Der}(\mathscr{G}, \mathscr{K})\left(U_{1} \cap U_{2} \cap U_{3} \cap U_{4}\right) \text {. }
$$

Then

$$
\left.\psi_{\alpha \beta \gamma}\right|_{U_{1} \cap U_{2} \cap U_{3} \cap U_{4}}=\varepsilon_{\alpha \beta}+\varepsilon_{\beta \gamma}+\varepsilon_{\gamma \alpha}
$$

and the result follows. 
The proof of the next theorem uses standard arguments and will be omitted.

THEOREM 4.5. The class of $\Delta(\xi)$ in $H^{2}(M, \operatorname{Der}(\mathscr{G}, \mathscr{K}))$ is independent of $(\xi)$. Denote this class by $c[M]$. For there to exist an imbedding $0 \rightarrow \mathscr{K} \rightarrow \mathscr{A} \rightarrow \mathscr{G} \rightarrow 0$ of $(M, \mathscr{G})$ in a supermanifold it is necessary and sufficient that $c[M]=0$. If $c[M]=0$ then the moduli space of such imbeddings is $H^{1}(M, \operatorname{Der}(\mathscr{G}, \mathscr{K}))$.

The next section gives some examples of these imbeddings.

5. Examples. 1. Let $B=\Lambda\left(\mathbf{R}^{L}\right), L \geqslant q . B^{p, q}$ has its standard supermanifold $\mathscr{A}=\hat{\mathscr{G}} \otimes \Lambda[q]$. Up to isomorphism there are no other supermanifolds over $B^{p, q}$. That is, $H^{1}\left(B^{p, q}, \operatorname{Der}(\mathscr{G}, \mathscr{K})\right)=0$.

To see this, let $\varepsilon: B^{p, q} \rightarrow \mathbf{R}^{p}$ be the natural projection. Let $U \subseteq \mathbf{R}^{p}$ be an open set and let $V=\varepsilon^{-1}(U)$. Let $C^{\infty}$ denote the sheaf of $C^{\infty}$ functions on $\mathbf{R}^{p}$ and let $\mathscr{A}^{\prime}=C^{\infty} \otimes B \otimes \Lambda[q]$. Then by equation (4.1), $\mathscr{A}=\varepsilon^{-1} \mathscr{A}^{\prime}$. By Lemma 4.1, $\mathscr{K}=$ $\varepsilon^{-1}\left(\mathscr{I}^{L+1} \otimes C^{\infty}\right)$, where $\mathscr{I}$ is the nilpotent ideal in $B \otimes \Lambda[q]$.

Lemma 5.1. $\delta$ induces an isomorphism $\operatorname{Der}\left(\mathscr{G}^{\infty}, \mathscr{K}\right) \simeq \operatorname{Der}(\mathscr{A}, \mathscr{K})$.

Proof. Define

$$
\operatorname{Der}\left(\mathscr{G}^{\infty}, \mathscr{K}\right) \rightarrow \operatorname{Der}(\mathscr{A}, \mathscr{K}), \quad X \rightarrow \bar{X}
$$

by $\bar{X} f=X \tilde{f}$. Since $\delta$ is surjective, this is injective. To see that it is surjective, take $Y \in \operatorname{Der}(\mathscr{A}, \mathscr{K})$ and set $Y=f^{i}\left(\partial / \partial \pi^{\prime}\right), f^{i} \in \mathscr{K}$. Now $\mathscr{K}=\mathscr{I}^{L+1}$ and $\left(\partial / \partial \pi^{i}\right)\left(\mathscr{I}^{L+1}\right) \subseteq \mathscr{I}^{L+1-\left|\pi^{i}\right|}$. Thus $Y(\mathscr{K}) \subseteq \mathscr{I}^{L+1} . \mathscr{I}^{L} \subseteq \mathscr{I}^{2 L+1}$ and $\mathscr{I}^{2 L+1}=0$ since $L \geqslant q$ and $\mathscr{I}^{L+q+1}=0$. Thus $Y=\bar{X}$ for some $X$.

Thus $\operatorname{Der}\left(\mathscr{G}^{\infty}, \mathscr{K}\right)$ is $\varepsilon^{-1}$ of a free $C^{\infty}$ module. The fiber of $\varepsilon^{-1}$ is $J^{p, q}$, where $J$ is the nilpotent ideal in $\Lambda\left(\mathbf{R}^{L}\right)$. So $H^{1}\left(V, \operatorname{Der}\left(\mathscr{G}^{\infty}, \mathscr{K}\right)\right)=H^{1}\left(U, C^{\infty}\right)^{n}$ for some $n$, and the right-hand side is 0 .

2. The second example involves the functor of points. This functor has been described by both Leites [9] and Batchelor [2]. Here is a review.

Let $(M, \mathscr{A}, \delta)$ be a supermanifold. If $(N, \mathscr{B}, \eta)$ is another supermanifold, a morphism $\Phi \in \operatorname{Hom}(\mathscr{B}, \mathscr{A})$ is a pair $\Phi=(\varphi, \tilde{\varphi})$ where $N \stackrel{\tilde{\varphi}}{\rightarrow} M$ is a $C^{\infty}(B)$ map, $\tilde{\varphi}^{-1} \mathscr{A} \stackrel{\varphi}{\rightarrow} \mathscr{B}$ is a homomorphism of sheaves of supercommutative algebras over $B$, and

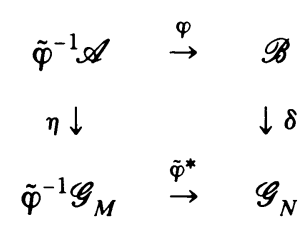

commutes.

If $N$ is a point, $\{\mathrm{pt}\}$, then $\mathscr{B}$ reduces to a single algebra $B \otimes \Lambda[n]$, and the evaluation map can be take to be $b \otimes \omega \stackrel{\varepsilon}{\rightarrow} b \varepsilon(\omega)$, where $\varepsilon: \Lambda[n] \rightarrow F$ is the familiar projection (i.e., one may take a coordinate system centered at pt). In this case the dimension of the supermanifold is $(0, n)$. With $B$ fixed, denote the $(0, n)$-dimensional supermanifold over $\{\mathrm{pt}\}$ by $n$. 
Consider the supermanifold morphisms, $\operatorname{Hom}(n, \mathscr{A})$ from $n$ to $\mathscr{A}$. For $h \in$ $\operatorname{Hom}(n, \mathscr{A})$, the map of topological spaces which underlies $h$ is simply a point $x \in M$, and in addition there is a $\mathbf{Z}_{2}$-graded algebra homomorphism

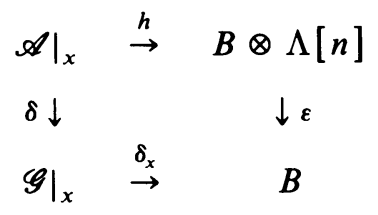

where the bottom arrow is evaluation at $x$.

There are maps $i: M \rightarrow \operatorname{Hom}(n, \mathscr{A})$ and $\sigma: \operatorname{Hom}(n, \mathscr{A}) \rightarrow M$ such that $\sigma \circ \iota=\mathrm{id}$. For $h \in \operatorname{Hom}(n, \mathscr{A})$, define

$$
\sigma(h)=\text { the image of pt. }
$$

For $x \in M$, define

$$
\iota(x) f=\tilde{f}(x) .
$$

Set $B_{n}=B \otimes \Lambda[n]$. Given an open set $U \subset M$ and given $f \in \mathscr{A}(U), f$ defines a function

$$
\bar{f}: \sigma^{-1}(U) \rightarrow B_{n}, \quad \bar{f}(h)=h(f) .
$$

Let $\xi^{1}, \ldots, \xi^{p+q}$ be a coordinate system for $\left.\mathscr{A}\right|_{U}$. For any $x \in U$ and $b \in \sigma^{-1}(x)$, let $\bar{\xi}(h)=\left(\xi^{1}(h), \ldots, \overline{\xi^{p+q}}(h)\right)$. Then $\bar{\xi}(h)$ lies in $B_{n}^{p, q}$ and $\varepsilon \circ \bar{\xi}=\tilde{\xi} \circ \sigma$ by assumption.

LemMA 5.2. Let $V=\tilde{\xi}(U) . \bar{\xi}$ carries $\sigma^{-1}(U)$ 1-1 onto $\varepsilon^{-1}(V)$.

Proof. $\xi$ carries $\left.\hat{\mathscr{G}} \otimes \Lambda[q]\right|_{V}$ isomorphically onto $\left.\mathscr{A}\right|_{U}$ so it suffices to show that $\bar{\pi}$ carries $\sigma^{-1}(V)$ 1-1 onto $\varepsilon^{-1}(V)$, where $\pi^{1}, \ldots, \pi^{p+q}$ is the standard coordinate system for $\hat{\mathscr{G}} \otimes \Lambda[q]$. Take $y \in \varepsilon^{-1}(V)$. Let $x=\varepsilon(y)$. Then $y=x+\left(c^{1}, \ldots, c^{p+q}\right)$, with each $c^{i}$ nilpotent. Consider the derivation $D_{y}=(-1)^{\left|\pi^{i}\right|} c^{i}\left(\partial / \partial \pi^{i}\right)$ acting on $\hat{\mathscr{G}} \otimes \Lambda[q] \otimes \Lambda[n]$. Define $\varepsilon^{-1}(V) \stackrel{\tau}{\rightarrow} \sigma^{-1}(V)$ by $\tau(y)=\delta_{x} \circ e^{D_{y}}$. Then

$$
\bar{\pi} \circ \bar{\tau}(y)=\left(\delta_{x} \circ e^{D_{y}}\left(\pi^{1}\right), \ldots, \delta_{x} \circ e^{D_{y}}\left(\pi^{p+q}\right)\right)=y .
$$

It remains to show that $\bar{\tau} \circ \bar{\pi}(h)=h$ for all $h \in \sigma^{-1}(V)$. Let $y=\bar{\pi}(h)=$ $\left(h\left(\pi^{1}\right), \ldots, h\left(\pi^{p+q}\right)\right)$. Let $\dot{x}=\varepsilon(y)$. Given $\left.f \in \hat{\mathscr{G}} \otimes \Lambda[q]\right|_{x}$, regard $f$ as an element of $\hat{\mathscr{G}} \otimes \Lambda[q] \otimes \Lambda[n]$. There exist $f_{1}, \ldots, f_{p+q} \in \hat{\mathscr{G}} \otimes \Lambda[q] \otimes \Lambda[n]$ such that $f=$ $f(y)+f_{i} \cdot\left(\pi^{i}-\pi^{i}(y)\right)$, where $f(y)$ is the polynomial continuation of $f$ to $y$. Then $f(y)=\delta_{x} \circ e^{D_{y}}(f)$, and $h(f)=f(y)=\bar{\tau} \circ \bar{\pi}(h)(f)$. So $h=\bar{\tau} \circ \bar{\pi}$ as desired.

One then verifies that if $\xi$ and $\psi$ are overlapping coordinate systems on $(M, \mathscr{A}, \delta)$, then $\bar{\psi} \circ \bar{\xi}^{-1}$ is a $C^{\infty}(B)$ diffeomorphism on a neighborhood in $B_{n}^{p, q}$, so that $\operatorname{Hom}(n, \mathscr{A})$ is a $C^{\infty}\left(B_{n}\right)$ manifold.

Suppose one starts with a Berezin-Kostant supermanifold. That is, $B=\mathbf{R}$ or $\mathbf{C}$. Then $\operatorname{Hom}(n, \mathscr{A})$ is a Rogers $G^{\infty}$ manifold based on $\Lambda[n]$. Let $\mathscr{G}_{n}$ be the sheaf of $C^{\infty}(\Lambda[n])$ functions on $\operatorname{Hom}(n, \mathscr{A})$. What is the space of supermanifold extensions of $\mathscr{G}_{n}$ ? Define

$$
\sigma^{-1} \mathscr{A} \otimes \Lambda[n] \stackrel{\delta_{n}}{\rightarrow} \mathscr{G}_{n}, \quad f \otimes \omega \rightarrow \bar{f} \cdot \omega \quad(\omega \text { is a scalar. })
$$

Let $\mathscr{A}_{n}=\sigma^{-1} \mathscr{A} \otimes \Lambda[n]$. 
Lemma 5.3. $\left(\operatorname{Hom}(n, \mathscr{A}), \mathscr{A}_{n}, \delta_{n}\right)$ is a supermanifold.

Proof. This is simply a restatement of equation (4.1).

Assume $n \geqslant q$. Let $\mathscr{K}_{n}=\operatorname{ker} \delta_{n}$. To see whether $\operatorname{Hom}(n, \mathscr{A})$ admits any supermanifold besides $\mathscr{A}_{n}$, consider $H^{1}\left(\operatorname{Hom}(n, \mathscr{A}), \operatorname{Der}\left(\mathscr{G}_{n}, \mathscr{K}_{n}\right)\right)$.

LEMMA 5.4. $\sigma^{-1} \mathscr{A}^{\delta_{n}} \rightarrow \mathscr{G}_{n}$ is injective.

Proof. By polynomial continuation, it suffices to establish that $\sigma^{-1} \mathscr{A}^{\delta_{n}} \mathscr{G}_{n}$ is injective along $M$, that is, that $\left.\mathscr{A} \stackrel{\delta_{n}}{\rightarrow} \mathscr{G}_{n}\right|_{M}$ is injective. Let $U$ be a neighborhood in $M$ and let $\xi$ be a coordinate system for $\left.\mathscr{A}\right|_{U}$, centered at $x \in U$. Let $\left.f \in \mathscr{A}\right|_{x}$, and assume $\delta_{n} f=0$. Then for all $\beta^{p+1}, \ldots, \beta^{p+q} \in \Lambda[n]_{1}$, and all $y$ near $x$, $\delta_{y} \circ e^{-\beta^{\prime}} \partial / \partial \xi^{i}(f)=0$. If $f=f_{\mu} \xi^{\mu}$ with $f_{\mu} \in \hat{\mathscr{A}}_{\xi}$, this says $f_{\mu}(y) \beta^{\mu}=0$ for all $y$ near $x$. Since $\Lambda[n]$ has more than $q$ algebraically independent elements, $f_{\mu}=0$ for all $\mu$.

The resemblance of the decomposition $\mathscr{A}_{n}=\sigma^{-1} \mathscr{A} \otimes \Lambda[n]$ to the decomposition of Theorem 1.2 is entirely superficial. $\operatorname{Hom}(n, \mathscr{A})$ is a $C^{\infty}(\Lambda[n])$ manifold, so that $\Lambda[n]$ is regarded not as the polynomial ring in the coordinate functions, but as the constant sheaf. $\operatorname{Hom}(n, \mathscr{A})$ has an additional structure not shared by general $C^{\infty}(\Lambda[n])$ manifolds. There is a distinguished sheaf of functions on $\operatorname{Hom}(n, \mathscr{A})$, namely $\sigma^{-1} \mathscr{A}$, which imbeds in $\mathscr{A}_{n}$.

$\sigma^{-1} \mathscr{A}$ can be described in a manner that emphasizes the derivations of $\mathscr{A}_{n}$. For the sake of discussion, suppose $B=\mathbf{R}$. $\operatorname{Hom}(n, \mathscr{A}), \mathscr{A}_{n}$ and $\operatorname{Der} \mathscr{A}_{n}$ all have distinguished subobjects: $\operatorname{Hom}(n, \mathscr{A})$ contains $M, \mathscr{A}_{n}$ contains $\sigma^{-1} \mathscr{A}$, and $\operatorname{Der} \mathscr{A}_{n}$ contains $\sigma^{-1}(\operatorname{Der} \mathscr{A})$, which acts on $\mathscr{A}_{n} \Lambda[n]$-linearly. The latter objects may be thought of as a real form of the former. Specifically, given $x \in \operatorname{Hom}(n, \mathscr{A})$ and $\left.f \in \mathscr{A}_{n}\right|_{x}, f$ continues to a section along $\sigma^{-1} \circ \sigma(x)$. Then $f$ is in $\sigma^{-1}(\mathscr{A})$ if and only if for all $X_{1}, \ldots, X_{k} \in \sigma^{-1}(\operatorname{Der} \mathscr{A}),\left.\overparen{X_{1} \circ \cdots \circ X_{k} f}\right|_{M}$ is real valued.

Let $\mathscr{N}$ denote the nilpotent ideal in $\mathscr{A}$. Let $J$ be the nilpotent ideal in $\Lambda[n]$. Then

$$
\mathscr{K}_{n}=\sum_{m=n+1-q}^{n} \sigma^{-1}\left(\mathscr{N}^{n+1-m}\right) \otimes J^{m} .
$$

Lemma 5.5. (1) $\operatorname{Der}\left(\sigma^{-1} \mathscr{A}, \mathscr{K}_{n}\right)=\mathscr{K}_{n} \otimes \sigma^{-1}(\mathscr{A}) \operatorname{Der}\left(\sigma^{-1} \mathscr{A}\right)$.

(2) $\operatorname{Der}\left(\mathscr{G}_{n}, \mathscr{K}_{n}\right)=\operatorname{Der}\left(\sigma^{-1} \mathscr{A}, \mathscr{K}_{n}\right)$.

Proof. For (1) simply write a $\mathscr{K}_{n}$-valued derivation as a linear combination of partial derivatives with $\mathscr{K}_{n}$ coefficients. (2) follows directly from Lemma 5.1.

It follows that

$$
\operatorname{Der}\left(\mathscr{G}_{n}, \mathscr{K}_{n}\right)=\sigma^{-1}\left[\sum_{m=n+1-q}^{n} \operatorname{Der}\left(\mathscr{A}, \mathscr{N}^{n+1-m}\right) \otimes J^{m}\right] .
$$

Now take a cover $\mathscr{U}=\left\{\left(U_{\alpha}, \xi_{\alpha}\right)\right\}$ of $M$ by coordinate neighborhoods for $\mathscr{A}$, such that for all $k>1$ and all $j>1$ and all $U_{1}, \ldots, U_{n} \in \mathscr{U}$,

$$
H^{k}\left(U_{1} \cap \cdots \cap U_{n}, \operatorname{Der}\left(\mathscr{A}, \mathscr{N}^{j}\right)\right)=0 \text {. }
$$


This can be done since $\mathscr{A}$ is locally isomorphic to a free $\mathscr{C}$ module, where $\mathscr{C}$ is the sheaf of smooth or holomorphic functions on $M$. ( $\mathscr{A}$ itself is not a sheaf of $\mathscr{C}$ modules.) Then as in Example 1,

$$
H^{k}\left(\sigma^{-1} U_{1} \cap \cdots \cap \sigma^{-1} U_{n}, \operatorname{Der}\left(\mathscr{G}_{n}, \mathscr{K}_{n}\right)\right)=0
$$

for all $U_{1}, \ldots, U_{n} \in \mathscr{U}$ and all $k>1$. Thus

$$
\begin{aligned}
H^{*}\left(\operatorname{Hom}(n, \mathscr{A}), \operatorname{Der}\left(\mathscr{G}_{n}, \mathscr{K}_{n}\right)\right)=H^{*}\left(\left\{\sigma^{-1} U_{\alpha}\right\}, \operatorname{Der}\left(\mathscr{G}_{n}, \mathscr{K}_{n}\right)\right) \\
=H^{*}\left(\left\{\sigma^{-1} U_{\alpha}\right\}, \sigma^{-1}\left(\sum_{m=n+1-q}^{n} \operatorname{Der}\left(\mathscr{A}, \mathscr{N}^{n+1-m}\right) \otimes J^{m}\right)\right) \\
=H^{*}\left(\left\{U_{\alpha}\right\}, \sum_{m} \operatorname{Der}\left(\mathscr{A}, \mathscr{N}^{n+1-m}\right) \otimes J^{m}\right) \\
=\sum_{m} H^{*}\left(M, \operatorname{Der}\left(\mathscr{A}, \mathscr{N}^{n+1-m}\right)\right) \otimes J^{m} .
\end{aligned}
$$

To summarize,

THEOREM 5.6. Let $(M, \mathscr{A})$ be a $(p, q)$-dimensional supermanifold with ground ring $F=\mathbf{R}$ or $\mathbf{C}$. Let $B=\Lambda\left(F^{n}\right)$. Then

1. $\operatorname{Hom}(n, \mathscr{A})$ is a $C^{\infty}(B)$ manifold.

2. $\left(\operatorname{Hom}(n, \mathscr{A}), \sigma^{-1} \mathscr{A} \otimes B, \delta_{n}\right)$ is a $C^{\infty}(B)$ supermanifold.

3. If $n \geqslant q$, the restriction of $\delta_{n}$ to $\sigma^{-1} \mathscr{A}$ is injective. That is, $\sigma^{-1} \mathscr{A}$ may be regarded as a sheaf of functions on $\operatorname{Hom}(n, \mathscr{A})$.

4. The supermanifold extensions of $\operatorname{Hom}(n, \mathscr{A})$ are classified by

$$
\sum_{m=n+1-q}^{n} H^{1}\left(M, \operatorname{Der}\left(\mathscr{A}, \mathscr{N}^{n+1-m}\right)\right) \otimes J^{m}
$$

where $J$ is the nilpotent ideal in $B$ and $\mathscr{N}$ is the nilpotent ideal in $\mathscr{A}$.

$\operatorname{Der}\left(\mathscr{A}, \mathscr{N}^{j}\right)$ is an $\mathscr{A}$ module, and in the $C^{\infty}$ category, $\mathscr{A}$ is fine. (See [8].) Thus,

Corollary 5.7. If $(M, \mathscr{A})$ is a $C^{\infty}$ Berezin-Kostant supermanifold, then $\operatorname{Hom}(n, \mathscr{A})$ admits a unique supermanifold compatible with its natural $C^{\infty}(B)$ structure.

In the holomorphic category, $\operatorname{Hom}(n, \mathscr{A})$ may admit many supermanifold extensions. For example, let $(M, \mathcal{O})$ be a complex manifold, and let $\Theta$ denote its holomorphic tangent sheaf. Let $\mathscr{E}$ be the trivial rank $q \mathcal{O}$-module. Then $(M, \Lambda \mathscr{E})$ is a Berezin-Kostant supermanifold, and its nilpotent ideal, $\mathscr{N}$, is $\sum_{j \geqslant 1} \Lambda^{j} \mathscr{E}$. Der $\mathscr{A}$ is simply $\Lambda \mathscr{E} \otimes\left(\mathscr{E}^{*} \otimes \Theta\right)$, so that $\operatorname{Der}(\mathscr{A}, \mathscr{N})=\mathcal{O}^{a} \oplus \Theta^{b}$ for some integers $a$ and $b$. Thus if either $H^{1}(M, \mathcal{O})$ or $H^{1}(M, \Theta)$ is nonzero, the supermanifold structure on $\operatorname{Hom}(n, \Lambda \mathscr{E})$ is nonunique.

\section{REFERENCES}

1. M. Batchelor, The structure of supermanifolds, Trans. Amer. Math. Soc. 253 (1979), 329-338.

2. __ Two approaches to supermanifolds, Trans. Amer. Math. Soc. 258 (1980), 257-270.

3. F. A. Berezin and D. A. Leites, Supermanifolds, Soviet Math. Dokl. 16 (1975), 1218-1221.

4. C. P. Boyer and S. Gitler, The theory of $\mathscr{G}^{\infty}$ supermanifolds, Trans. Amer. Math. Soc. 285 (1984), 241-267. 
5. B. S. DeWitt, Supermanifolds, Cambridge Univ. Press, Cambridge, 1984.

6. K. Gawedzki, Supersymmetries-mathematics of supergeometry, Ann. Inst. H. Poincaré, Sect. A 27 (1977), 335-366.

7. A. Jadczyk and K. Pilch, Superspaces and supersymmetries, Comm. Math. Phys. 78 (1980), 373-390.

8. B. Kostant, Graded manifolds, graded Lie theory, and prequantization, Differential Geometric Methods in Mathematical Physics (Proc. Sympos. Univ. Bonn, Bonn, 1975), Lecture Notes in Math., vol. 570, Springer-Verlag, Berlin, 1977, pp. 177-306.

9. D. A. Leites, Introduction to supermanifold theory (preprint).

10. J. M. Rabin and L. Crane, Global properties of supermanifolds, Comm. Math. Phys. 100 (1985), 141-160.

11. A. Rogers, A global theory of supermanifolds, J. Math. Phys. 21 (1980), 1352-1365.

12. M. Rothstein, Deformations of complex supermanifolds, Proc. Amer. Math. Soc. 95 (1985), 255-260.

Department of Mathematics, University of Washington, Seattle, Washington 98195

Current address: School of Mathematics, Institute for Advanced Study, Princeton, New Jersey 08540 